DRAFT VERSION OCTOBER 9, 2018

Preprint typeset using $\mathrm{LT}_{\mathrm{E}} \mathrm{X}$ style emulateapj v. 6/22/04

\title{
ROCKING THE LIGHTHOUSE: CIRCUMPULSAR ASTEROIDS AND RADIO INTERMITTENCY
}

\author{
J. M. CORDES \& R. M. SHANNON \\ Astronomy Department, Cornell University, Ithaca, NY 14853
}

Draft version October 9, 2018

\begin{abstract}
We propose that neutral, circumpulsar debris entering the light cylinder can account for many time-dependent pulsar phenomena that are otherwise difficult to explain. Neutral material avoids propeller ejection and injects sufficient charges - after heating, evaporation, and ionization - to alter current flows and pair-production and thus trigger, detune, or extinguish coherent emission. Relevant phenomena, with time scales from seconds to months, include nulls, rotating radio transients (RRATs), rapid changes in pulse profile ("mode changes"), variable subpulse drift rates, quasi-periodic bursts from B1931+24, and torque variations. Over the $\sim 10^{7} \mathrm{yr}$ lifetime of a canonical pulsar with $10^{12} \mathrm{G}$ surface magnetic field, less than $10^{22} B_{12} \mathrm{~g}\left(\lesssim 10^{-6} M_{\oplus}\right)$ is needed to modulate the Goldreich-Julian current by $100 \%$. Circumpulsar material originates from metal-rich, supernova fallback gas that aggregates into asteroids. Debris disks can inject sufficient material on time scales of interest, yet be too tenuous to form large planets detectable in pulse timing data. Asteroid migration results from collisions and the radiation-driven Yarkovsky and Poynting-Robertson effects. For B1931+24, an asteroid in a $\sim 40$ day elliptical orbit pollutes the magnetosphere stochastically through collisions with other debris. Injection is less likely for hot, young and highly magnetized pulsars or millisecond pulsars that pre-ionize any debris material well outside their small magnetospheres. Injection effects will therefore be most prominent in long-period, cooler pulsars, consistent with the distribution of relevant objects in $P$ and $\dot{P}$. A pulsar's spin history and its radiation-beam orientation may influence whether it displays nulling, RRATs and other effects. Subject headings: stars: neutron — pulsars: general — pulsars:individual (B1931+24, B0656+14) — accretion - acceleration of particles
\end{abstract}

\section{INTRODUCTION}

Rotation-driven pulsars display myriad phenomena in their radio emission on time scales that range from nanoseconds to years. Here we consider a unifying model for phenomena that occur on time scales from pulse-to-pulse to monthlike or longer, including: (1) null pulses, where the emission evidently vanishes but returns in pulses that maintain synchronism with the spin; (2) drifting subpulses, whose systematic variations in pulse phase appear to be related to circulation of emission regions around the magnetic pole; (3) mode changes, where the pulse shape changes discontinuously between two or more shapes, often accompanied by dramatic changes in flux density; and (4) rotating radio transients (RRATs) that correspond to bursts of single or a few pulses spaced by very long intervals (minutes to hours) (McLaughlin et al. 2006). A few objects show giant pulses that can be $\geq 10^{3}$ larger in amplitude than the average pulse (e.g. Cognard et al. 1996; Hankins et al. 2003; Johnston \& Romani 2003; Cordes et al. 2004; Cairns 2004).

Coherent emission wanes on time scales $\sim 10^{7} \mathrm{yr}$ for canonical pulsars - those with surface magnetic fields $\sim$ $10^{12} \mathrm{G}$ - and on much longer times for millisecond pulsars (MSPs; $10^{8}-10^{9} \mathrm{G}$ ). We assume, of course, that the spindown time scale, $\tau_{s}=P / 2 \dot{P}$, is a reasonable, but by no means perfect, proxy for chronological age. The distribution of pulsars in the period-period derivative $(P-\dot{P})$ diagram shown in Figure 1 displays a dearth of pulsars with $\dot{P}<10^{-16.7} \mathrm{~s} \mathrm{~s}^{-1} P^{3}$ in the bottom right corner that reflects these time scales. However, it is not clear whether pulsars shut off their radio emission according to a threshold effect, such as the inability to sustain electron-positron cascades whose flows induce coherent emission, or that the rotational energy loss rate, $\dot{E}=I \Omega \dot{\Omega}$, simply cannot provide sufficient power to drive the radio beam luminosity (Arzoumanian. Chernoff \& Cordes 2002). Empirically, the latter seems favored because the density of pulsars in the $P-\dot{P}$ diagram rolls off smoothly rather than sharply at a "cliff". However, pair cascades may terminate differently in pulsars with the same $P$ and $\dot{P}$ if surface magnetic fields show object-dependent complexity, thus smearing out any cliff effect.

In this paper we develop a mechanism that pertains to the four phenomena described above but is particularly focused on nulling pulsars and RRATs. Both show episodes of radio quiesence that appear to be totally devoid of any radio emission to levels $\lesssim 10^{-2}$ of their 'on' states. Off states can last for long time periods (from minutes to days), although some nulling pulsars are off only for a few pulse periods. Both classes of objects are largely found on the right-hand periphery of the population of canonical pulsars in the $P-\dot{P}$ diagram (Figure 1). As pointed out by many (e.g. Biggs 1992), nulling may signify a faltering emission process that signifies incipient complete shut off of radio emission. Instead, we suggest that nulling and RRATs are both caused by pollution of NS magnetospheres by low-level accretion from a circumpulsar asteroid disk. This idea is motivated by the fact that one nulling pulsar, B1931+24, has state durations of days that are cyclical with a $\sim 40$-day quasi-period (Kramer et al. 2006). In addition, early work by Cheng (1985) explored the role played by interstellar grains in the current flows of active pulsars and their manifestation on the spindown rates of pulsars. Our model differs by considering material that originates in a reservoir of orbiting debris, most likely supernova fallback material that has formed a low-mass asteroid belt. Orbits around neutron stars provide a plethora of time scales that can be imprinted on electrodynamic processes by material injected into the magnetosphere. Michel \& Dessler (1981) considered plasma disks around pulsars to address issues of elec- 
trodynamics in pulsar magnetospheres. Here too, our model is different in considering a largely neutral disk comprising macroscopic objects.

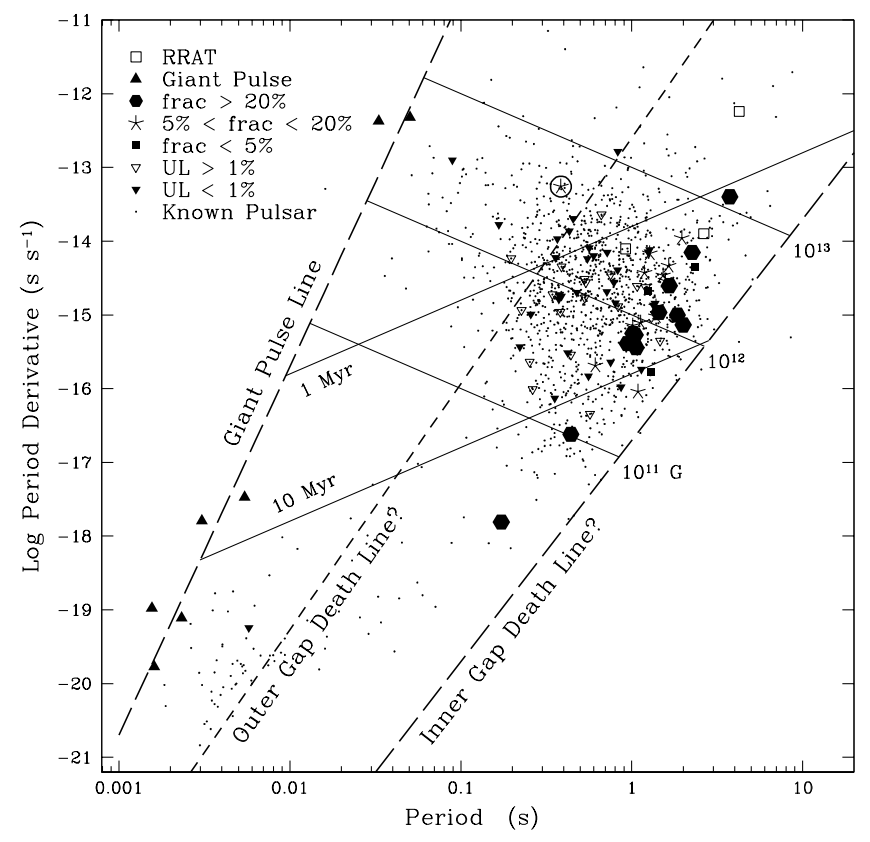

FIG. 1.- The period-period-derivative diagram for pulsars. Rotating radio transients (RRATs), pulsars that show giant pulses, and nulling pulsars that display different nulling fractions are distinctly labelled (see legend). Points labelled "UL" indicate pulsars for which upper limits have been placed on the nulling fraction. Solid lines of constant magnetic field and lines of constant characteristic spindown age, $P / 2 \dot{P}$ are shown. The most leftward dashed line denotes constant magnetic field strength at the light cylinder (with a field strength equal to that of the Crab pulsar) that seems to be a trademark of objects showing giant pulses (e.g. Cognard et al. 1996). The inner-gap "death line" is drawn as the rightward dashed line at an arbitrary location that is consistent with the diminishment of the radio pulsar density in the diagram. The middle dashed line is an outer-gap death line from Takata et al. 2006). The pulsar B0656+14 is circled because it is an object with high surface temperature that shows low-level nulling.

Cheng's (1985) grain inflow model was developed to explain an apparent correlation between $P \dot{P}$ and space velocity that was identified in the 1980s. While that correlation is now thought to be insignificant for canonical pulsars ${ }^{1}$, extrinsic charges from inflowing grains also solved the issue of how a NS avoids being charged up by self-generated current flows and it also obviated the need for self-sustained pair production. However, the grain model appears to work only for cool $\left(\sim 10^{5} \mathrm{~K}\right)$, long-period pulsars because grains are evaporated by thermal radiation at distances of $10^{9}-10^{10} \mathrm{~cm}$, comparable to the light-cylinder radii of long-period pulsars, but much larger than those of short-period and, presumbably, younger and hotter pulsars. For our purposes, the longer-period and (presumably) cooler NS are just the sample we wish to address. In many respects, Cheng's analysis also applies to grains delivered from asteroids that are evaporated and ionized inside the magnetosphere. The differences are that (a)

\footnotetext{
${ }^{1}$ Note that space velocities are much less for pulsars that have undergone accretion-induced spinup and consequent diminishment of the dipole field component. However, there does not appear to be a correlation between $B_{\text {dipole }}^{2} \propto P \dot{P}$ and space velocity among pulsars that have not accreted much material.
}

orbital delivery is expected to be episodic, as opposed to the steady flow of interstellar grains; (b) asteroids and rocks will penetrate further into the magnetosphere than the $\sim 0.1 \mu \mathrm{m}$ grains considered by Cheng; and (c) circumpulsar disks probably vary widely from pulsar to pulsar, providing a natural explanation for why pulsars with very similar spin parameters $(P, \dot{P})$ are dramatically different with respect to the occurrence of nulls, etc.

In the next sections, we describe relevant phenomena and key elements of the model for producing nulls, RRATs and other bursts, including (a) formation of the circumpulsar disk; (b) delivery of asteroids from the disk to the magnetosphere; (c) tidal disruption, evaporation, and ionization; and (d) effects on gap accelerators and radio emission. We also discuss implications for spin variations of NS from the torque fluctuations that are expected and we suggest possible tests for the model.

\section{SALIENT PROPERTIES OF NULLS AND RRATS}

The most striking feature of both nulls and RRATs is the rapid transition between states, either on to off or vice versa. These transitions appear to occur in less than one spin period in many cases (e.g. Deich et al. 1986) and are thus too fast to be caused by propagation effects in the interstellar medium (ISM). The nulling phenomenon includes pulsars that are mostly on and occasionally show nulls with durations of a few spin periods and others where the pulsar is mostly in the off state for periods of up to days. In a few well studied objects, a slow roll-off of the pulsed flux precedes a null. In one case (B1944+17; Deich et al. (1986)) the slow decay is over a few pulse periods and in another, a few tens of periods (J1752+2359; Lewandowski et al. (2004)). Nulling objects occur throughout the grouping of canonical pulsars in the $P-\dot{P}$ diagram. However, those with null fractions $>20 \%$ are strikingly more common near the right-hand side of the grouping. Notable outliers exist, such as B0656+14, as labelled in Figure 11 Data used to form Figure 1 are given in Table A1 and are from multiple sources in the literature and also from the ATNF/Jodrell pulsar catalog (http://www.atnf.csiro.au/research/pulsar/psrcat/)

Some objects with nearly the same period and period derivative null while others do not. This suggests that $P$ and $\dot{P}$ are not the sole determinants of a pulsar's instantaneous radio luminosity. The surface magnetic field strength is commonly estimated to be $B_{12}=\left(P \dot{P}_{-15}\right)^{1 / 2}$ (with $\left.\dot{P}=10^{-15} \dot{P}_{-15}\right)$ and numerous work has associated either the pseudo-luminosity $S D^{2}$, where $S$ is the flux density averaged over period and $D$ is the distance in $\mathrm{kpc}$, or the true beam luminosity (e.g. Arzoumanian. Chernoff \& Cordes 2002) with a power-law model $P^{x} \dot{P}^{y}$, with exponents $x, y$ determined from some fitting process. The presence and absence of nulling objects with the same spin parameters suggests that other variables are involved. Intrinsic models might include instabilities in the current flow that are regulated by the thermal time constant of the NS crust (Cheng \& Ruderman 1980; Jones 1982) or incomplete filling of the open field line region with emission regions associated with drifting subpulses (e.g. Deshpande \& Rankin 1999).

Figure 2 shows the nulling fraction plotted against the angle between the spin axis and the magnetic axis $\alpha$ for the known nulling pulsars. Spin-magnetic axis angles were compiled from the analyses of Rankin (1990, 1993), Everett \& Weisberg (2001), and Mitra \& Rankin (2002). 
With the exception of $\mathrm{B} 2315+21$, which has a nulling fraction of $3.0 \pm 0.5 \%$ (Biggs 1992), all the nulling pulsars have a spin axis-magnetic axis offset of at most $60^{\circ}$.

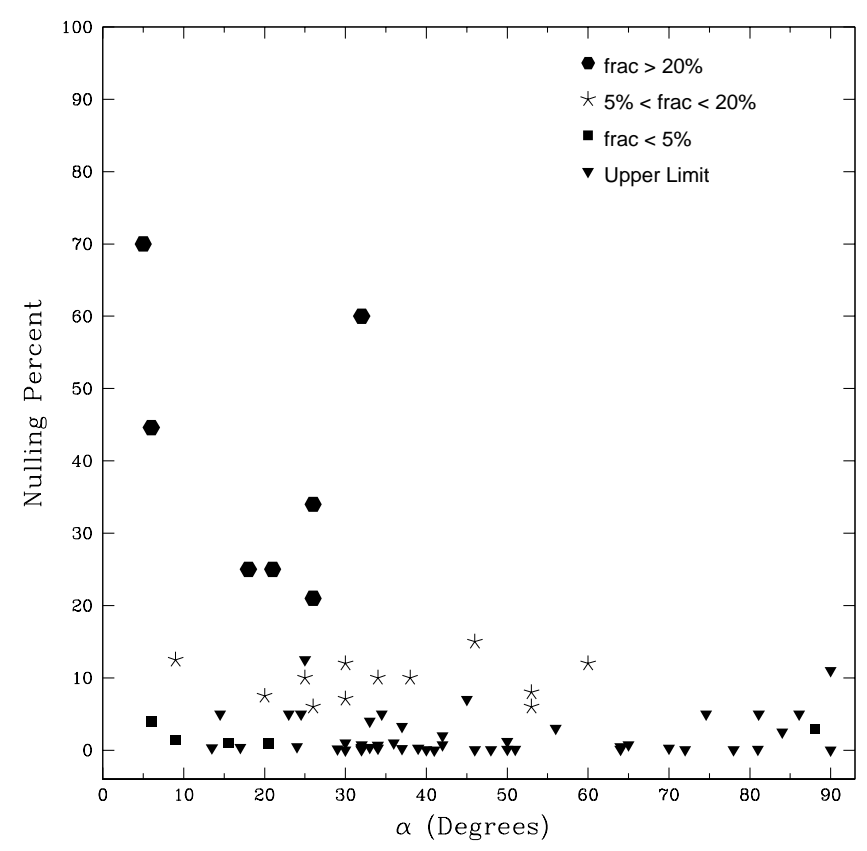

FIG. 2.- Pulsar nulling fraction as a function of inclination angle $\alpha$ between the magnetic and spin axes. The model presented in this paper suggests that orthogonally rotating $\left(\alpha \approx 90^{\circ}\right)$ would be less likely to null, as the beam would severely disrupt asteroid transport into the magnetosphere, which is consistent with these observations.

RRATs are perhaps an extreme form of nulling. Pulse event rates range from 0.3 to $20 \mathrm{hr}^{-1}$ in the eleven objects reported by McLaughlin et al. (2006). A twelfth object (Cordes et al. 2006) has a much higher rate $\left(\sim 200 \mathrm{hr}^{-1}\right)$ was also discovered as an aperiodic source but later found to be periodic.

RRAT pulses typically occur singly, in contrast to nulling pulsars in their on states. Though RRATs are biased to long periods, as with nulls there is a short-period example. The RRAT Galactic population is probably very large in spite of the small, known sample because their low duty cycles imply a small probability of detection and because the Parkes multibeam survey covered only a narrow range of Galactic latitude. Non-pulsed X-ray emission has been detected from the RRAT J1819-1458 (Reynolds et al. 2006), which has the largest apparent magnetic field $\left(5 \times 10^{13} \mathrm{G}\right)$, and has the lowest spindown age (117 kyr) of the 11 RRATs.

\section{BASIC PICTURE}

The essential feature of our model is a disk of asteroids that is sustained in largely neutral form for tens of millions of years from which asteroids or their fragments are injected into the magnetosphere of a NS. To account for observed nulls and RRATs, injection must be episodic but, over long time periods, statistically homogeneous. Thus, our model differs substantially from that of Cheng (1985), who considered steady injection of grains from the general ISM, requiring their survival through the bow shocks that we know are associated with essentially all pulsars, owing to their large space velocities, $V_{\mathrm{psr}} \sim 10^{2}-10^{3} \mathrm{~km} \mathrm{~s}^{-1}$. Moreover, his model implies that pulsar radio emission should always be steady (or always quenched) rather than intermittent. Cheng's model may be relevant for older, cooler neutron stars that have no debris disks around them. Our model also differs substantially from that of Colgate \& Petschek (1981), who considered interstellar objects that would reach the NS surface in producing gamma-ray bursts from Galactic NS.

A supernova fallback disk (see below) is metal rich and can be sufficiently low mass $\left(10^{-6} M_{\odot}\right)$ or compact (inside the tidal disruption radius) to prevent formation of massive planets that would be detectable individually in pulsar timing measurements. However, rocks can form that will resist tidal shredding until they are well inside the magnetospheres of longperiod pulsars and will stay neutral until they are melted and ionized by radiation from the NS surface or magnetosphere. Delivery of rocks to the magnetosphere can occur from collisions and orbital perturbations by protoplanets if the disk extends beyond the tidal disruption radius. Alternatively, rock migration from a compact disk will occur from the radiation driven Yarkovsky effect.

The situation we identify is essentially the complement of that needed to account for planet formation around ordinary stars. Planet formation requires that planetesimals exist outside the gravitational tidal radius $r_{\mathrm{tg}} \sim 10^{11} \mathrm{~cm}$ (see below) so that runaway accretion followed by oligarchic growth and mergers of oligarchs can form planetary core masses of Earth mass or larger (e.g. Rafikov 2003; Goldreich et al. 2004). The planet building process takes only $\sim 10^{5} \mathrm{yr}$ in the inner solar system but cleanup of debris can take several hundred million years (Goldreich et al. 2004). For our case, the disk need not be stable; in fact it can disperse on a time scale $\sim 10-100$ Myr, the apparent radio-emitting lifetime of a canonical pulsar.

Once a disk becomes neutral, asteroid migration will follow from many of the processes that have been identified in the solar system. These include formation and growth of planetesimals through collisions, destructive collisions, tidal circularization and spin alignment, shepherding by larger bodies, injection into resonances, heating from photon and particle fluxes and subsequent anisotropic re-radiation, and heating from induction and tidal flexure. Once injected into the magnetosphere of a pulsar, asteroids will evaporate and, when ionized, provide charges that are subject to strong electromagnetic forces. Such forces are most prominent in "gaps" where there is a potential drop along magnetic field lines that will accelerate charges inward and outward. Even though the structure of such gaps has been a subject of analysis for more than three decades, a definitive picture has yet to emerge. However, the prominence of pulsars at both radio frequencies and in high-energy bands attests to the fact that such gaps exist. In Figure 3 we show a schematic magnetosphere for an object with a spin-magnetic-moment angle $\alpha=20^{\circ}$. The gaps shown are schematic representations that are consistent with those in the literature but may underestimate their true extent if gaps extend all the way to the inner gap. It is plausible for the geometry shown that asteroidal material that penetrates the LC will be subject to gap potentials.

Injected charges are accelerated in gap electric fields and can activate a quiescent region, such as an outer-gap, as a source of gamma-rays that pair produce, or they can temporarily shut off the pair production of an active gap by reducing the accelerating field. A pair avalanche from an outer-gap (as opposed to a sustained cascade) can influence other regions in the magnetosphere, as suggested by Wright (2003) and 
Zhang, Gil \& Dyks (2006) for self-driven processes within the magnetosphere rather than by extrinsic causes, as we argue here.

In order for this picture to be viable, it must satisfy three constraints: (1) the disk must be sufficiently low-mass that it not produce planets that would be detectable in pulse timing measurements or by other means; (2) the rate of mass inflow from the disk into the LC must be large enough to influence magnetospheric current flows on time scales of interest; and (3) the disk must survive long enough (e.g. $>10 \mathrm{Myr}$ ) to influence the electrodynamics of old pulsars. Next we set limits on the properties of the disk with these constraints in mind.

\subsection{Observational Constraints on Disk Masses}

Infrared observations with Spitzer and previous instruments (e.g. Bryden et. al. 2006, and references therein) have failed to detect disk emission around the MSP B1257+12, which harbors at least three planets (Konacki \& Wolszczan 2003). Nonetheless, the observations still allow a debris disk around the MSP consisting of (e.g.) $100-\mathrm{km}$ asteroids with as much as $10^{-2} M_{\oplus}$ total mass. Related theoretical work (Miller \& Hamilton 2001; Bryden et. al. 2006) imply that asteroids larger than $\sim 1 \mathrm{~km}$ can exist for longer than $1 \mathrm{Gyr}$ around the MSP.

Phillips (1993) placed limits on asteroidal masses around the Vela pulsar from an upper limit on the unpulsed flux $S_{u}$ that would be produced by reflections of the pulsed radio flux $S_{p}$ from an asteroid belt. His limit for a power-law size distribution of slope -3.5 extending from $R_{a 2}=100 \mathrm{~km}$ down to $R_{a 1}$ equal to the $20 \mathrm{~cm}$ radio wavelength is

$$
M_{a} \lesssim 53 M_{\oplus} \frac{r_{\mathrm{AU}}^{2}}{A_{0.1}}\left(\frac{S_{u} / S_{p}}{2 \times 10^{-4}}\right)\left(\frac{\rho}{3 g c m^{-3}}\right)\left(\frac{\sqrt{R_{a 1} R_{a 2}}}{141 m}\right)
$$

For compact disks with $r_{\mathrm{AU}} \sim 10^{-2}$, this result becomes modestly constraining as $M_{a} \lesssim 10^{-2.3} M_{\oplus}$ but does not limit the mass in rocks that are smaller than the $20 \mathrm{~cm}$ wavelength.

Pulsar timing measurements are sensitive to orbital perturbations from objects of about the Moon's mass (Konacki \& Wolszczan 2003). The rms sinusoidal timing residual caused by a single object of mass $m_{a}$ in a circular orbit with period $P_{\text {orb }}$ is

$$
\delta t \approx \frac{a \sin i m_{a}}{\sqrt{2} c M_{*}} \approx 0.85 \mathrm{~ms} \sin i P_{\mathrm{orb}}^{2 / 3}\left(\frac{m_{a}}{M_{\oplus}}\right)\left(\frac{1.4 M_{\odot}}{M_{\odot}}\right) .
$$

Limits on single planet masses depend on data set lengths but are typically (Thorsett et al. 1993) $\sim 1 M_{\oplus}$ for $P_{\text {orb }} \sim 1$ yr. An ensemble of $N_{a}$ asteroids in a belt with total mass $M_{a}$ with rms semi-major axis $a_{\mathrm{rms}}$ yields an rms timing residual

$$
\delta t_{\mathrm{rms}}=0.76 \mathrm{~ms} \frac{\zeta a_{\mathrm{rms}} \sin i}{\sqrt{N_{a}}}\left(\frac{M_{a}}{M_{\oplus}}\right),
$$

where $a_{\mathrm{rms}}$ is in $\mathrm{AU}$ and $\zeta \approx 2$ is the ratio of rms to mean asteroidal mass. $^{2}$

Timing residuals for long-period pulsars are typically in the range of 0.1 to $1 \mathrm{~ms}$ (rms) and show a variety of characteristic time scales as well as displaying non-stationary behavior (i.e. rms residual that depends on data span length). The limit on the total mass in an asteriod belt is therefore

$$
M_{a} / M_{\oplus} \lesssim(0.1-1) \times \frac{\sqrt{N_{a}}}{a_{\mathrm{rms}} \sin i}
$$

${ }^{2}$ For a pulsar timing data set of length $T$, only orbital periods $\lesssim 2 \pi T$ should be included in the calculation of $\delta t_{\text {rms }}$ because a low-order polynomial that is fitted and removed filters out the contributions of longer-period objects.
Thus disks of $\gg 1 M_{\oplus}$ aggregate mass in $N_{a} \gg 1$ asteroids can easily reside around many pulsars, particularly if $a_{\text {rms }} \ll 1 \mathrm{AU}$, as we consider here. In addition, such disks can contribute significantly to the timing noise while not yielding detection of any individual asteroid. To detect at the $\ell \times \sigma$ level the sinusoidal variation in a Fourier analysis of $n_{d}=n_{d, 3} 10^{3}$ data points with white noise measurement errors of $\sigma_{w, 1} \mathrm{~ms}$, an asteroid would have to satisfy $a_{\mathrm{AU}} \sin i\left(m_{a} / M_{\oplus}\right) \gtrsim 0.04 \ell \sigma_{w, 1} n_{d, 3}^{-1 / 2}$. In the case where timing residuals are dominated by asteroidal noise from $N_{a}$ asteroids, to be detectable an asteroid must have a mass that is a multiple of the mean asteroidal mass, $\left\langle m_{a}\right\rangle M_{a} / N_{a}$, of

$$
m_{\mathrm{a}, \max } /\left\langle m_{a}\right\rangle \gtrsim \ell \zeta\left(a_{\mathrm{rms}} / a_{\max }\right) \sqrt{N_{a} / n_{d}}
$$

which is large for a large number of asteroids and reasonable values of other parameters (e.g. $\zeta=2, a_{\mathrm{rms}} / a_{\max }=1 / 2$, and $n_{d} \approx 10^{3}$ ). We conclude that canonical pulsars can harbor asteroid disks with a sizable fraction of an Earth mass in the disk and show timing noise consistent with that measured. As shown below, very low mass asteroid disks are sufficient to disrupt the magnetospheres of these same pulsars.

\subsection{Magnetospheric Currents and Disk Lifetime}

To see if a disk can provide mass at interesting rates after $10 \mathrm{Myr}$, we consider the current and implied mass rate needed to alter the electric field in a magnetosphere. In order for the acceleration region to exist, it is necessary for the magnetosphere to have low density.

The fiducial charge density in such objects is of order the Goldreich-Julian density (Goldreich \& Julian 1969), $\rho_{e}=$ $-\Omega \cdot B / 2 \pi c$, corresponding to a number density

$$
n_{\mathrm{GJ}}(r)=\frac{|\Omega \cdot B(r)|}{2 \pi Z e c} \approx 10^{10.8} \mathrm{~cm}^{-3}\left(\frac{|\cos \psi| B_{12}}{Z P}\right)\left(\frac{r}{R_{*}}\right)^{-3}
$$

Here $Z$ is the charge per particle, $\psi$ is the local angle between the spin axis and dipolar field, and $R_{*}$ is the NS radius.

The current from the magnetic polar cap (defined as the footprint of dipolar field lines that do not close within the LC) with area $A_{\mathrm{PC}}$ is (in charges s${ }^{-1}$ )

$$
\dot{N}_{\mathrm{GJ}}=c n_{\mathrm{GJ}} A_{\mathrm{PC}}=\frac{2 \pi^{2} B R^{3}}{Z c e P^{2}}=10^{30.14} \mathrm{~s}^{-1} Z^{-1} B_{12} R_{6}^{3} P^{-2} .
$$

For $\mu_{p}$ proton masses per charge, the corresponding mass rate is

$$
\dot{M}_{\mathrm{GJ}}=10^{6.3} \mathrm{~g} \mathrm{~s}^{-1} \mu_{p} B_{12} R_{6}^{3} P^{-2},
$$

only a fraction $10^{-11.6}$ of the Eddington mass accretion rate for a NS. Note that the entire magnetosphere has a minimum mass $M_{\text {mag }} \approx 4 \pi B_{\text {surface }} R_{*}^{3} \mu_{p} m_{p} \ln \left(r_{\mathrm{LC}} / R_{*}\right) / e c P \approx$ $10^{7} \mathrm{~g} B_{12} \mu_{p} P^{-1}$.

For a pulsar born with a $10 \mathrm{~ms}$ spin period that spins down to $10 \mathrm{~s}$ in $10^{7} \mathrm{yr}$, the total mass that would have to be accreted to account for the GJ current is, if we assume constant magnetic field,

$$
M_{\mathrm{GJ}}=10^{21.9} \mathrm{~g} \mu_{p} B_{12} R_{6}^{3} \tau_{7}\left(\frac{P_{0}}{10 \mathrm{~ms}} \frac{P_{\tau}}{10 \mathrm{~s}}\right)^{-1},
$$

which is equivalent to the mass in a $\sim 100 \mathrm{~km}$ sized asteroid. (Michel \& Dessler 1981) made a similar point in their discussion of much more massive plasma disks around pulsars. A pulsar cannot accrete during approximately the first $10^{5} \mathrm{yr}$ of 


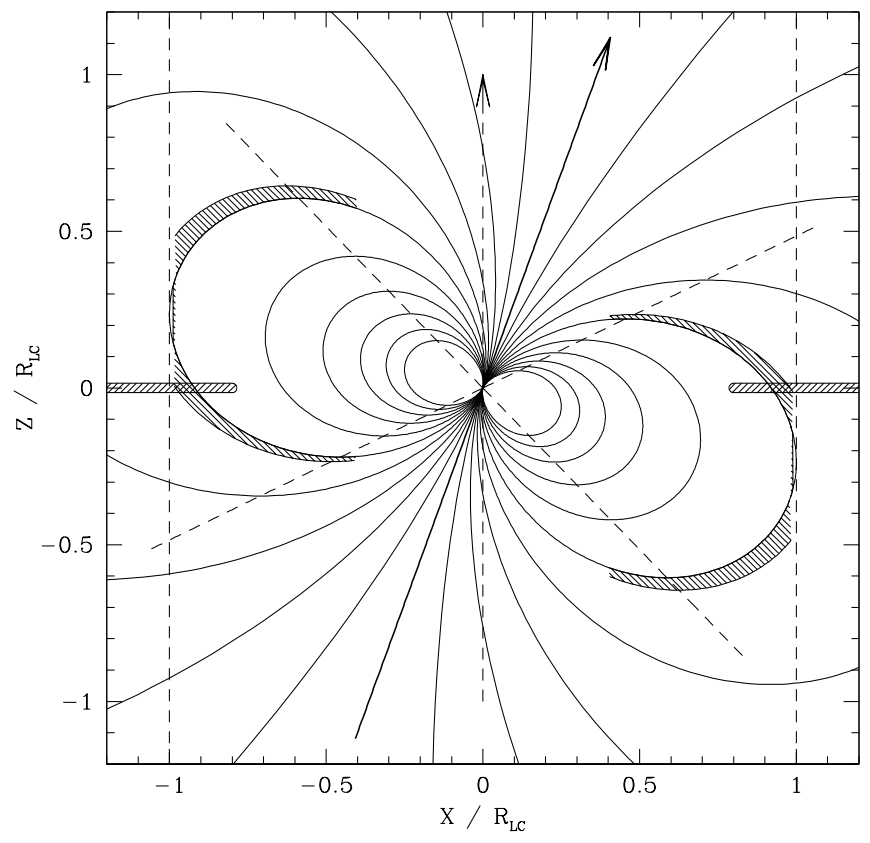

FIG. 3.- The magnetosphere of a pulsar showing the locations of outergap acceleration regions where $E_{\|} \neq 0$. The asteroid belt is shown (shaded) in the $z=0$ plane. The other shaded regions show outer gaps whose extents along and transverse to the last closed field line within the light cylinder (LC) are arbitrary but consistent with discussion in the literature (e.g. Takata et al. 2006). These gaps may extend all the way from the LC (denoted as vertical dashed lines near the horizontal boundaries of the diagram) to the NS surface, and the inner gap (too small to be shown). The spin axis is the arrowed (vertical) dashed line and the magnetic axis is shown by a arrowed (slanted) solid line. Length scales are in units of the LC radius.

its life because tidal shredding and thermal radiation will ionize material before it can enter the magnetosphere. However, a pulsar spends most of its lifetime at long periods, so our estimate is relevant.

Consider a disk that contains a total mass $M_{a}$ in $N_{a}$ asteroids within minimum and maximum radii, $r_{1}, r_{2} \gg r_{1}$, and thickness $2 H \equiv \epsilon_{h} r_{2}$. Inward migration implies a lifetime (for fixed $\left.N_{a}\right)$

$$
\tau_{d}=\frac{M_{a}}{\dot{M}_{a}}=\frac{N_{a}}{\dot{N}_{a}} .
$$

The flux of asteroids through a cylinder centered on the NS with fiducial radius $r$ and surface area $A_{c}=2 \pi \epsilon_{h} r_{2} r$ is $\dot{N}_{a}=$ $n_{a} A_{c}|\dot{r}| \approx 2 N_{\mathrm{a}} r|\dot{r}| r_{2}^{-2}$, implying a disk lifetime that must be larger than the radio lifetime $\tau_{\text {psr }}$. This yields the constraint

$$
\frac{2 r|\dot{r}|}{r_{2}^{2}}<\tau_{\mathrm{psr}}^{-1}
$$

In order to provide at least a fraction $\epsilon_{\mathrm{GJ}}$ of the GoldreichJulian mass rate, we require $\dot{M}_{a} \geq \epsilon_{\mathrm{GJ}} \dot{M}_{\mathrm{GJ}}$, where $\dot{M}_{a}=\dot{N}_{a} m_{a}$ and $m_{a}$ is a typical asteroid mass, or

$$
\frac{2 r|\dot{r}|}{r_{2}^{2}}>\frac{\epsilon_{\mathrm{GJ}} \dot{M}_{\mathrm{GJ}}}{M_{a}}
$$

Evaluating the two constraints assuming, while taking $\tau_{\mathrm{psr}}=$ $100 \mathrm{Myr}$ ), we find

$$
10^{-21.5} \mathrm{~s}^{-1} \frac{\epsilon_{\mathrm{GJ}} \mu_{p} B_{12} R_{*, 6}^{3}}{P^{2}\left(M_{a} / M_{\oplus}\right)}<\frac{2 r|\dot{r}|}{r_{2}^{2}}<10^{-15.5} \mathrm{~s}^{-1} \tau_{\mathrm{psr}, 100}^{-1}
$$

where we have assumed uniform mass density $\rho$ in an asteroid. It is obvious that a disk can easily satisfy these constraints and not be detectable in pulse timing data. The implied migration rate $\dot{r}$ into a cylinder with fiducial radius $r=10^{10} r_{10} \mathrm{~cm}$ from a disk of outer radius $r_{2}=10^{-2} r_{2,2}$ AU must satisfy

$$
\begin{array}{rl}
10^{-9.1} & \mathrm{AU} \mathrm{Myr}^{-1} \epsilon_{\mathrm{GJ}} \mu_{p} B_{12} R_{*, 6}^{3} P^{-2}\left(\frac{M_{a}}{M_{\oplus}}\right)^{-1} r_{2,2}^{2} r_{10}^{-1}<|\dot{r}| \\
& <10^{-3.1} \mathrm{AU} \mathrm{Myr}^{-1} r_{2,2}^{2} r_{10}^{-1} \tau_{\mathrm{psr}, 100}^{-1} .
\end{array}
$$

Influx rates much larger than these occur in the solar system due to a variety of effects that we consider below.

\section{CIRCUMPULSAR DISKS}

A dust disk around the anomalous X-ray pulsar 4U 0142+61, a magnetar, has recently been detected with Spitzer Space Telescope and is estimated to have mass $\sim 10 M_{\oplus}$ and a lifetime of at least 1 Myr (Wang. Chakrabarty \& Kaplan 2006). While other direct evidence for circumpulsar disks is lacking, there are several lines of argument that support their existence - perhaps transitory - around at least some NS. The strongest is the presence of planets around at least two NS, one an MSP in the Galactic disk (B1257+12) and the other in a globular cluster (B1620-26, Sigurdsson et al. 2003). For these cases, disks probably arose from ablative disruption of a companion star and are not relevant to the model proposed here, which concerns canonical pulsars that are now isolated. Michel \& Dessler (1981) explored how the electrodynamics of pulsars are altered by fossil disks and could alleviate the need for the then "extreme" magnetic field strengths of $10^{12} \mathrm{G}$ as well as account for some of the observational properties of pulsars, including nulling. The disks proposed by Michel \& Dessler (1981) are substantially more massive (up to $10^{-4} M_{\odot} \approx 33 M_{\oplus}$ ) than those considered here.

Fallback disks are plausible features of supernova explosions because of the wide range of energy and angular momentum carried by material that is initially outward-moving. Simulations indicate that the fallback disk around a NS typically does not provide the conditions needed for planet building, primarily because it is born compact and does not spread much in radius when it becomes neutral and essentially inviscid (Menou et al. 2001). Rocks with large tensile strengths can grow from the metal-rich gas and survive well inside the Roche radius, $r_{\text {tg }}$ (see below).

Menou et al. (2001) and Lu \& Cheng (2002) consider metal-rich, supernova fallback disks of mass $10^{-6}-10^{-3} M_{\odot}$. After a phase of relatively strong accretion onto the NS that mimics that in binary systems and produces X-ray emission, the disk becomes neutral on a time scale $\sim 10^{3}-10^{4} \mathrm{yr}$. The disks are born very compact $\left(\sim 10^{8}-10^{9} \mathrm{~cm}\right)$ and spread to $10^{9}-10^{10} \mathrm{~cm}$ by the time they become neutral (Menou et al. 2001). Eksi \& Alpar (2005) argue that disks are not disrupted by pulsar radiation pressure and may extend into the light cylinder. Blackman \& Perna (2004) discuss modification of the spindown law by an accretion disk and fit models to the Crab and Vela pulsars' braking indices and spindown ages to constrain disk parameters. Jiang \& Li (2005) consider how accretion from fallback disks with masses of $10^{-6}-10^{-2} M_{\odot}$ will significantly spin down NS during their first $10^{4} \mathrm{yr}$, thus providing a mechanism for "injection" of pulsars into the Galactic populatation over a range of periods that extends to $>0.1 \mathrm{~s}$ (e.g. Vivekanand \& Naravan 1981).

Growth of planetesimals in the disk depends on whether the material is interior or exterior to the tidal disruption ra- 
dius, which is, for a self-gravitating object with mass density $\rho$ orbiting a $1.4 M_{\odot} \mathrm{NS}$,

$$
r_{\mathrm{tg}} \approx\left(\frac{3 M_{*}}{2 \pi \rho}\right)^{1 / 3} \sim 10^{11} \rho^{-1 / 3} \mathrm{~cm} .
$$

The tidal radius is well outside the light cylinder radius,

$$
r_{\mathrm{LC}}=c / \Omega=10^{9.7} \mathrm{Pcm},
$$

for all known radio pulsars but is comparable to the light cylinders of magnetars with $P \sim 10$ s. The spin rates of solarsystem asteroids indicate that they are mostly rubble piles with negligible tensile strength (Richardson et al. 2002) but asteroids around pulsars may be stronger macroscopic objects if they are metal rich and fractionated and thus can remain intact well inside the gravitational tidal disruption radius. A meter-sized rock, $R_{a}=10^{2} R_{2} \mathrm{~cm}$, with tensile strength $\mathcal{T}=10^{8} \mathcal{T}_{8}$ dyn $\mathrm{cm}^{-2}$, is tidally disrupted at

$$
r_{\mathrm{tm}} \approx\left(\frac{G M_{*} \rho R_{a}^{2}}{\mathcal{T}}\right)^{1 / 3} \approx 10^{7.4} \mathrm{~cm}\left(\rho R_{2}^{2} \mathcal{T}_{8}^{-1}\right)^{1 / 3}
$$

which is about 27 times the NS radius, $R_{*} \approx 10 \mathrm{~km}$, similar to altitudes where coherent radio emission is produced (Dvks et al. 2004). Thus, should any large $\sim 10 \mathrm{~km}$-sized planetesimals exist in the disk, they will tidally shred near the LCs of 1-s pulsars, while meter-sized rocks can penetrate deeply into the magnetosphere until they evaporate.

\section{HEATING OF ASTEROIDS}

Heating of asteroids will ultimately evaporate grains and allow them to be ionized efficiently by radiation from the NS and magnetosphere. Cheng (1985) concluded that $\sim 0.1 \mu \mathrm{m}$ iron and silicate grains reach distances $\sim 1-3 \times 10^{9} T_{5} \mathrm{~cm}$. Depending on their sizes and inward velocities, meter (or larger) sized rocks will reach similar radii before they are evaporated.

The equilibrium temperature of an asteriod determines whether or not it can enter the magnetosphere intact and in a largely neutral state. The temperature of a low-albedo asteroid in thermal equilibrium with surface radiation from the NS is

$$
T_{a, *}=T_{*}\left(\frac{R_{*}}{2 r}\right)^{1 / 2} \approx 707 K T_{5} r_{10}^{-1 / 2},
$$

where $T_{*}$ is the effective radiation temperature that ranges from $\sim 10^{5}$ to a few times $10^{6} \mathrm{~K}$. Cooling mechanisms are not well identified and recent work (Slane et al. 2002) yields an upper limit of $10^{6} \mathrm{~K}$ for the young NS 3C58 while some older pulsars show higher temperatures. Moreover, NS mass determinations now show a broader range, extending from 1.3 $M_{\odot}$ to as high as $2.1 M_{\odot}$ (Nice et al. 2005), which signifies that a broad range of surface temperatures should be expected for objects of the same age (Yakovlev \& Pethick 2004) because cooling is much more efficient for high-mass NS.

Heating from magnetospheric radiation is mostly by beamed X-rays with luminosity $L_{X} \approx 10^{-3} \dot{E}$ (Becker \& Trümper 1997),

$$
T_{a, m} \approx 612 K\left(g_{b} I_{45} \dot{P}_{-15} P^{-3}\right)^{1 / 4}\left(\frac{L_{x} / \dot{E}}{10^{-3}}\right)^{1 / 4} r_{10}^{-1 / 2},
$$

where the beaming factor $g_{b} \approx\left(8 / \pi \theta_{b}\right)^{1 / 4}>1$ if the beam of size $\theta_{b}(\mathrm{FWHM})$ illuminates the asteriod and zero if it does not.
An additional source of heating is ohmic dissipation (induction heating), discussed in the Appendix. The dominant effect is from current flow between the asteroid and magnetosphere. The regime is different from the induction heating of asteriods in the solar nebula during the Sun's T-Tauri phase (e.g. Sonett et al. 1970; Herbert 1989), where the ambient plasma density in a much stronger (than present) solar wind provides a current circuit through the asteroid that causes ohmic heating. In the pulsar case, the asteriod is in a near vacuum, at least near the light cylinder, so charge carriers are available primarily from the asteriod itself. In moving closer to the NS, evaporation from radiative heating becomes significant (see below), providing a large number of charges. During this phase, runaway ohmic heating will occur (R. V. E. Lovelace, private communication) even if, as in the solar nebula case, ohmic heating is limited by diagmagnetism from current-induced magnetic fields (Sonett et al. 1970). At $2000 \mathrm{~K}$, which an asteroid reaches at $r \sim 10^{9} \mathrm{~cm}$ around a $10^{5} \mathrm{~K}$ and $10^{12} \mathrm{G} \mathrm{NS}$, evaporation begins to provide sufficient charges for significant ohmic heating, leading to runaway heating that causes the asteroid to explode. Lower magnetic fields allow the asteroid to reach slightly smaller radii before exploding. Perhaps the only way to get asteroids to significantly smaller radii is for them to intrude along the spin axis of pulsars with nearly aligned magnetic moments. The minimum radius reached is perhaps irrelevant as long as charged particles are injected inside the magnetosphere where they are electromagnetically captured.

Finally, tidal heating of an object can occur if its orbit is elliptical (or if it inspirals quickly), as occurs with solar-system objects such as Io, Europa and Enceladus (p. 171 Murrrav \& Dermott 1999). Peale. Cassen \& Revnolds (1979) give the heating rate as

$$
\frac{d E}{d t}=\frac{36 \pi}{19} \frac{e^{2} \rho^{2}}{Q \mu}\left(\frac{R_{a}}{r}\right)^{7} \frac{\left(G M_{*}\right)^{5 / 2}}{r^{1 / 2}},
$$

where $\mu$ is the rigidity and $Q$ is the specific dissipation rate (loss of energy per orbit). The implied radiative equilibrium surface temperature is

$$
T_{\mathrm{a}, \mathrm{t}}=10^{-1.85} K\left(\frac{(e \rho)^{2}}{Q \mu_{12}}\right)^{1 / 4} R_{2}^{5 / 4} r_{10}^{-15 / 8},
$$

where $\mu_{12}=10^{-12} \mu$. Tidal heating increases faster with decreasing $r$ than other heating processes and could be quite large for kilometer-sized objects that reach $10^{8} \mathrm{~cm}$; however, tidal disruption occurs before this takes place. Thus, unless the rigidity is small, tidal heating is at most secondary to the other heating mechanims.

To summarize, radiative heating of asteroids will dominate other processes for very hot NS or those with large magnetospheric luminosities beamed toward the asteroid belt. For these objects, entry into the magnetosphere will be prevented because material is ionized outside the LC. For other objects, some combination of radiation and induction heating will evaporate asteroids at radii of order $10^{9} \mathrm{~cm}$, well inside the LCs of many objects. Thus we expect that objects that can accrete significant amounts of asteroidal material into their LCs will occupy certain regions of the $P-\dot{P}$ diagram that correspond to objects with different ages, LC sizes, and ability to heat incoming objects.

For a fixed value of $T_{a}$ at the LC radius, a scaling law for $\dot{P}$ vs. $P$ can be calculated. Using the spindown age, $\tau_{s}=P / 2 \dot{P}$, 
as a proxy for chronological age, we find $\dot{P} \propto P^{5}$ for magnetospheric or induction heating and $\dot{P} \propto P^{1+1 / 2 q}$ for heating from thermal radiation whose temperature scales as $\tau_{s}^{-q}$. Cooling curves show a wide range of $q$, but for objects $\sim 10^{5}$ yr old, $q \sim 0.14$ while at ages longer than about $10^{6} \mathrm{yr}$, surface temperatures fall off steeply, corresponding to $q \sim 0.4$ to 1 (e.g. Figure 1 of Yakovlev \& Pethick 2004). For a slow falloff with age, $q \approx 0.14, \dot{P} \propto P^{4.6}$. Thus heating by magnetospheric radiation or by thermal NS radiation (in intermediate age objects) will yield lines of constant asteroidal temperature at the light cylinder distance from the NS that scale roughly as $\dot{P} \propto P^{5}$, which are steeper than the putative inner-gap death line. However, older pulsars will fall on a locus with $\dot{P} \propto P^{1.5}$ to $P^{2.3}$.

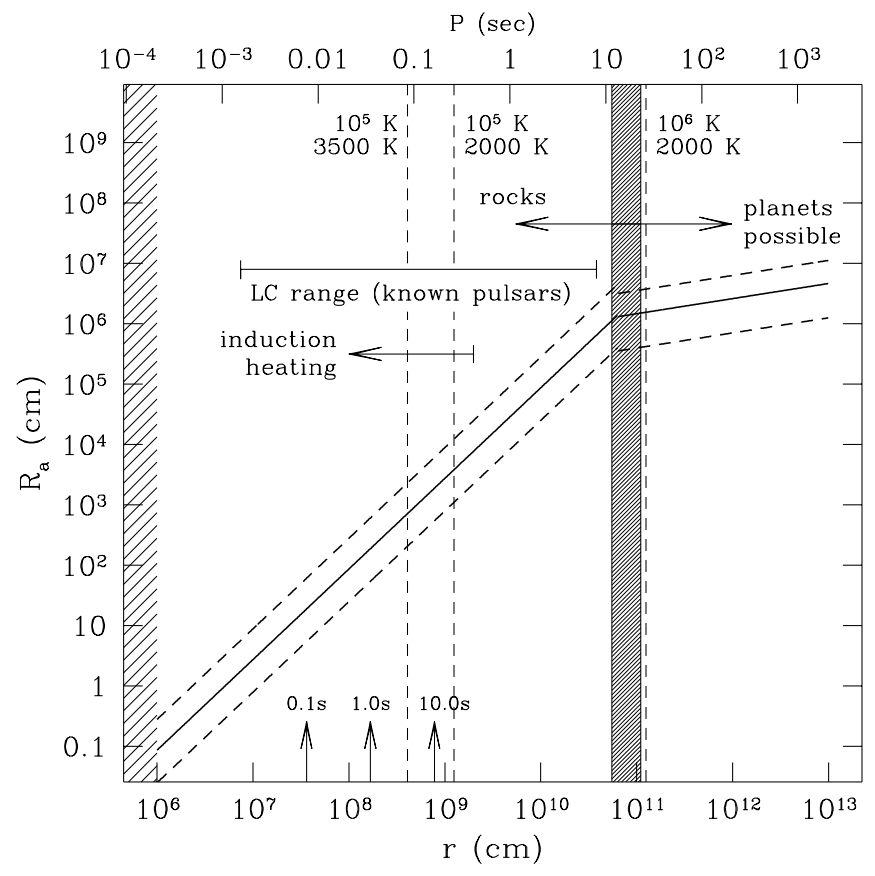

FIG. 4.- Asteroid radius, $R_{a}$, plotted against distance from the NS, $r$. The top horizontal scale is the period for which the light-cylinder radius $r_{\mathrm{LC}}=c P / 2 \pi$ matches the radius on the bottom horizontal scale and the horizontal bar designates the range of $\mathrm{LC}$ radii for known pulsars. The shaded region at the left-hand edge designates the inside of the neutron star. For nominal parameters, tidal disruption of gravitationally bound objects occurs at $r_{\mathrm{tg}} \sim 10^{11} \mathrm{~cm}$ (vertical shaded region). At larger distances, planets may form while material closer to the pulsar will only form rocks held together by their tensile strength. The leftward going arrow indicates where induction heating starts to be important for pulsars with canonical magnetic field strengths. Slanted curves to the left of $r_{\text {tg }}$ designate rock sizes that can be held together by tensile strengths of $10^{5}$ and $10^{8} \mathrm{dyn} \mathrm{cm}^{-2}$ at densities of 8 and $1 \mathrm{gm} \mathrm{cm}^{-3}$, respectively. The slanted curves to the right are the sizes of planetesimals that undergo runaway growth in an annulus of width twice their Hills radius in the circumpulsar disk. Vertical dashed lines designate radii at which rocks will reach $2000 \mathrm{~K}$ for a NS surface temperature of $10^{6} \mathrm{~K}$ and $2000 \mathrm{~K}$ or 3500 $\mathrm{K}$ at $10^{5} \mathrm{~K}$. Upward going arrows designate the radii at which an orbiting object co-rotates with the NS for spin periods of $0.1,1$ and $10 \mathrm{~s}$. Parameters include disk surface densities of $\Sigma=1$ to $20 \mathrm{~g} \mathrm{~cm}^{-2}\left(r / 10^{-2} \mathrm{AU}\right)^{-3 / 2}$ for internal asteroid densities $\rho=8$ to $1 \mathrm{~g} \mathrm{~cm}^{-3}$.

\section{MIGRATION OF DISK MATERIAL INTO THE MAGNETOSPHERE}

Disks can supply inward moving asteroids, as we show here, that stay neutral until they enter the LCs of cooler and longer-period pulsars at rates that are consistent with those for nulls and RRATs. While inward migration from radiative effects can occur rather slowly for a given object, once it enters the magnetosphere, we assume that ionized material from the asteroids will be subjected to electric forces that drastically alters its trajectory. Alternatively, collisions in the disk, which occur at interesting rates even for low-mass disks, provide more direct injection trajectories into the magnetosphere.

Studies of accretion onto compact objects usually consider ionized gas, which is subject to Lorentz forces that lead to the requirement that material needs to orbit faster than the magnetosphere at the Alfvén radius in order for it to be accreted. Slowly orbiting material is flung outward by the propeller effect (Illarionov \& Sunvaev 1975), resulting in spindown of the star. The situation is different if the material is neutral. An uncharged rock (or one with small charge-to-mass ratio) will not respond to the magnetic field. However, its surface charge and polarizability will lead to an interaction. These effects are small for macroscopic (meter-sized) objects. The main consideration is the set of forces on the rock as it evaporates and becomes ionized. High-melting temperature material can persist to radii of $10^{9.1} \mathrm{~cm}$ at $2000 \mathrm{~K}$ and $10^{8.6} \mathrm{~cm}$ at $3500 \mathrm{~K}$ around a NS with surface temperature of $10^{5} \mathrm{~K}$ (Figure 4). These melting-point radii are similar to those at which the rock will co-rotate with the NS, as indicated for periods of 0.1, 1 and 10s in Figure 4 By the time the rock is ionized, which is probably outside the corotation radius, $r_{c o}=\left[G M_{*} P^{2} /(2 \pi)^{2}\right]^{1 / 3} \approx 10^{8.2} \mathrm{~cm} P^{2 / 3}$, for most pulsars, it encounters regions in the magnetosphere where $E_{\|} \neq 0$ and is electrically captured.

\subsection{Nominal Disk Parameters}

Consider an asteroid disk of total mass $M_{a}$ with minimum and maximum radii, $r_{1}, r_{2} \gg r_{1}$, and thickness $2 H \equiv \epsilon_{h} r_{2}$ yielding a volume $V_{d} \approx(\pi / 2) \epsilon_{h} r_{2}^{3}$, average surface density

$$
\bar{\Sigma}=\frac{M_{a}}{\pi\left(r_{2}^{2}-r_{1}^{2}\right)}
$$

and an optical depth

$$
\tau=\frac{3}{4} \frac{\bar{\Sigma}}{\rho \tilde{R}_{a}}
$$

where $\tilde{R}_{a}$ is a characteristic asteroid size and $\rho$ is its internal mass density. For a distribution of asteroid sizes, $\tilde{R}_{a}=$ $\left\langle R_{a}^{3}\right\rangle /\left\langle R_{a}^{2}\right\rangle$ which is, for a power-law asteroid size distribution, $f\left(R_{a}\right) \propto R_{a}^{-m}, R_{a 1} \leq R_{a} \leq R_{a 2}$ with an equilibrium distribution $(m=7 / 2)$ (Dohnanvi 1969), $\tilde{R}_{a}=\sqrt{R_{a 1} R_{a 2}}$. The mean time between collisions is

$$
t_{c}=\frac{2 H}{\tau v_{\mathrm{rms}}}
$$

where $v_{\text {rms }}$ is the rms random velocity. The characteristic spreading time for the disk due to collisions only is (p. 496 Murrrav \& Dermott 1999)

$$
t_{\text {coll }}=\frac{1}{\tau \Omega_{\text {orb }}}\left(\frac{r_{2}-r_{1}}{\tilde{R}_{a}}\right)^{2} .
$$

For nominal parameter values $r_{1} \ll r_{2}, r_{2}=10^{-2} r_{2,-2} \mathrm{AU}$, $\tilde{R}_{a}=1 R_{\mathrm{a}, \mathrm{km}} \mathrm{km}$, and $M_{a}=10^{-4} M_{a,-4} M_{\oplus}$, we have the following typical values for the asteroid disk:

$$
\begin{aligned}
\bar{\Sigma} & =8.5 \mathrm{~g} \mathrm{~cm}^{-2} M_{a,-4} r_{2,-2}^{-2} \\
\tau & =10^{-4.2}\left(\rho \tilde{R}_{\mathrm{a}, \mathrm{km}}\right)^{-1} M_{a,-4} r_{2,-2}^{-2}
\end{aligned}
$$




$$
\begin{aligned}
\Omega_{\mathrm{orb}} & =10^{-1.9} \mathrm{~s}^{-1} r_{10}^{-3 / 2} M_{*, 1.4}^{1 / 2} \\
t_{\mathrm{coll}} & =10^{3.2} \mathrm{Gyr} \rho \tilde{R}_{\mathrm{a}, \mathrm{km}}^{-1} r_{2,-2}^{11 / 2} M_{\mathrm{a},-4}^{-1} M_{*, 1.4}^{-1 / 2} \\
t_{c} & =10^{2.9} \mathrm{yr} \epsilon_{h} \rho v_{\mathrm{rms}}^{-1} r_{2,-2}^{3} \tilde{R}_{\mathrm{a}, \mathrm{km}} M_{\mathrm{a},-4}^{-1} \\
\epsilon_{h} & =\frac{2 v_{\mathrm{z}, \mathrm{rms}}}{\Omega_{\mathrm{orb}} r_{2}}=10^{-4.0} v_{\mathrm{z}, \mathrm{rms}} M_{*, 1.4}^{-1 / 2} r_{10}^{3 / 2} r_{2,-2}^{-1} \\
N_{a} & =10^{8.2} M_{\mathrm{a},-4}\left(\rho \tilde{R}_{\mathrm{a}, \mathrm{km}}^{3}\right)^{-1} .
\end{aligned}
$$

where $v_{\text {rms }}$ and $v_{z, r m s}$ are rms velocities in $\mathrm{km} \mathrm{s}^{-1}$, with the $\mathrm{z}$ direction parallel to the disk's axis. We have evaluated $t_{\text {coll }}$ using an orbital frequency corresponding to an orbital radius, $r_{2} / 2$. In these and other calculations we ignore general relativistic corrections to orbital parameters, though apsidal advance and Lense-Thirring precession will be significant for orbits near or inside the LC. However, these effects will likely be subdominant to the much stronger electromagnetic forces in the region of strong gravity (and strong electromagnetic field) close to the surfaces of the NS .

\subsection{Debris Migration}

Collisions can direct some asteroids or their fragments into the magnetosphere. However, additional processes now known to be significant in the solar system are likely to also be important in the circumpulsar disk. These include the diurnal and seasonal Yarkovsky effects for larger rocks $(\sim 1$ $\mathrm{m})$ and the Poynting-Robertson effect for small grains (e.g. Burns et al. 1979).

The diurnal Yarkovsky effect results from the net force on an illuminated object whose afternoon surface is hotter than its nighttime surface. It can lead to an increase or decrease in semi-major axis depending on the lag angle of the hottest surface. The seasonal Yarkovsky effect applies to objects with spin axes tilted from their orbital plane, causing a net force along the spin axis that always decreases the semimajor orbital radius. In the solar system the diurnal effect is larger than the seasonal effect. Poynting-Robertson acceleration also decreases the orbital radius and is smaller than the Yarkovsky effect by a factor $v_{a} / c$, all else being equal, but is important for small grains that are isothermal for which the Yarkovsky effect vanishes. Near-Earth objects can migrate up to $10^{-3} \mathrm{AU} \mathrm{Myr}^{-1}$ at $1 \mathrm{AU}$ from the Sun from the seasonal Yarkovsky effect, which we take as a fiducial value.

The Poynting-Robertson effect drags small particles into the Sun on a time scale $t_{\mathrm{PR}}=4 \pi \rho c^{2} r^{2} R / 3 L \approx$ $672 \mathrm{yr} \rho R_{-4} r_{\mathrm{AU}}^{2} L_{\odot} / L$. NS thermal radiation yields a time scale for grains of size $10^{-4} R_{-4} \mathrm{~cm}$ at $10^{10} r_{10} \mathrm{~cm}$ (e.g. Rybicki \& Lightman 1986, p. 153)

$$
t_{\mathrm{PR}}=16.8 \mathrm{yr} \frac{\rho R_{-4} r_{10}^{2}}{R_{*, 6}^{2} T_{*, 5}^{4}}
$$

For nominal disk parameters, where disk-wide collisions are frequent, a steady supply of grains will be injected into the magnetosphere, similar to the interstellar flow proposed by Cheng (1985).

We now focus on the seasonal Yarkovsky effect because it provides a viable lower bound on the rate of inflow into the LCs of some pulsars. The Yarkovsky effect has been detected directly through measured accelerations of asteroids using radar techniques(Cheslev et al. 2003; Nesvorný \& Bottke 2004). It plays a key role in asteroid migration, especially to Earth-crossing orbits, by moving objects in the asteroid belt into mean-motion resonances that then cause much faster orbital evolution. The seasonal Yarkovsky effect causes a secular change in semi-major axis of the form

$$
\dot{r} \propto-\frac{(1-A) L}{c \rho R_{\mathrm{a}} \Omega_{\mathrm{orb}} r^{2}}\left(\frac{\Delta T}{T}\right)
$$

where $L$ is the luminosity, $\rho$ and $A$ are the mass density and albedo of the asteriod, and $\Delta T / T$ is the fractional temperature difference caused by misalignment of the spin axis from the normal to the orbital (or invariant) plane (Rubincam 1998). The above proportionality can be derived by assuming simplistically that a spherical asteroid has a temperature enhancement $\Delta T$ for the hemisphere facing the direction of motion. At $r=1 \mathrm{AU}, \dot{r} \approx-10^{-3} \mathrm{AU} \mathrm{Myr}^{-1}$ for a basaltic asteroid of $100 \mathrm{~m}$ size; values of $\dot{r}$ for iron asteroids are smaller by about a factor of two. The effect asymptotes to zero for smaller asteroids as they trend toward isothermality and is smaller for more massive asteroids.

The penetration depth for thermal X-rays $\ell_{X} \sim 0.1-1 \mu \mathrm{m}$ (Cheng 1985; Voit 1991) for 0.1 to $1 \mathrm{keV}$ photons, so macroscopic objects will be heated asymmetrically. The thermal skin depth for iron with thermal conductivity $\kappa=4 \times$ $10^{6} \mathrm{erg} \mathrm{s}^{-1} \mathrm{~cm}^{-1} \mathrm{~K}^{-1}$, heat capacity $C_{p}=5 \times 10^{6} \mathrm{erg} \mathrm{g}^{-1} \mathrm{~K}^{-1}$ and density $\rho=8 \mathrm{~g} \mathrm{~cm}^{-3}$ is

$$
\ell_{s}=\left(\frac{\kappa}{\rho \Omega_{\mathrm{orb}} C_{p}}\right)^{1 / 2} \approx 27 \mathrm{~cm}\left(\frac{1.4 M_{\odot}}{M_{*}}\right)^{1 / 4} r_{10}^{3 / 4} .
$$

Basalt and other materials have substantially smaller thermal conductivities yielding 3 times smaller skin depths, so conceivable materials can sustain temperature gradients to distances near the LC if they are larger than a meter.

We suppose that rocks possess spins with random orientations owing to collisions or chaotic dynamics, so any heating will drive the seasonal Yarkovsky effect. In a pulsar disk $\sim 10^{-3}$ AU from the NS, the Yarkovsky effect will be larger than for the solar system for fixed luminosity, but we must take into account both the thermal radiation from the NS and non-thermal radiation from the magnetosphere. For a thermal luminosity $L \approx 10^{28.9} \mathrm{erg} \mathrm{s}^{-1} R_{*, 6}^{2} T_{*, 5}^{4}$, the Yarkovsky drift rate will be

$$
\dot{r} \approx-10^{-7.2} \mathrm{AUMyr}^{-1}\left(\frac{\left|\dot{\mathrm{r}}_{\mathrm{ss}, 1 \mathrm{AU}}\right|}{10^{-3} \mathrm{AU} \mathrm{Myr}^{-1}}\right) \frac{R_{*, 6}^{2} T_{*, 5}^{4}}{r_{10}^{1 / 2} R_{\mathrm{a}, \mathrm{km}}},
$$

which is much smaller than the reference solar-system rate we have adopted, $\left|\dot{r}_{\mathrm{ss}, 1 \mathrm{AU}}\right| \approx 10^{-3} \mathrm{AU} \mathrm{Myr}^{-1}$, owing to the small thermal luminosity and the much larger orbital frequency.

Now we consider the fraction of the pulsar's spindown energy loss rate, $\dot{E}$, that is in a form that can heat a planetesimal. The LC is at the transition from the near zone to wave zone for rotational energy losses and most of the spindown loss rate, $\dot{E}$, is in low-frequency waves that will have little effect on a rock's dynamics. Cheng (1985) concluded that relativistic particles from the pulsar wind heat grains negligibly while X-rays dominate the ionization of grains after they enter the magnetosphere, a result that also applies to the heating of grains and rocks in the current model. Typically the nonthermal X-ray luminosity of a pulsar satisfies $L_{X} \approx 10^{-3} \dot{E}$, so

$$
\begin{aligned}
& \dot{r} \approx-10^{-5.5} \mathrm{AUMyr}^{-1} \times \\
& \quad\left(\frac{\mid \dot{r}_{\mathrm{ss}, 1 \mathrm{AU} \mid}}{10^{-3} \mathrm{AU} \mathrm{Myr}^{-1}}\right) g_{b} r_{10}^{-1 / 2} R_{\mathrm{a}, \mathrm{km}}^{-1}\left(\frac{L_{X} / \dot{E}}{10^{-3}}\right)\left(\frac{\dot{E}}{L_{\odot}}\right)
\end{aligned}
$$


where we have used the beaming factor $g_{b}$ defined before, which can exceed unity if the beam illuminates the asteroid but vanishes if not. Thus the Yarkovsky drift rate can be much larger for nonthermal radiation than for thermal radiation.

\subsection{Asteroid flux into the light cylinder \\ 6.3.1. Yarkovsky Migration}

The flux of asteroids through a cylinder centered on the NS with fiducial radius $r$ and surface area $A_{c}=2 \pi \epsilon_{h} r_{2} r$ is $\dot{N}_{a}=$ $n_{a} A_{c}|\dot{r}| \approx 2 N_{\mathrm{a}} r \dot{r} r_{2}^{-2}$, or

$$
\begin{aligned}
\dot{N}_{a} \approx & \frac{3}{2 \pi} \frac{M_{a}}{\rho \tilde{R}_{a}^{3}} \frac{r|\dot{r}|}{r_{2}^{2}} \approx \frac{3}{\pi} \bar{\Sigma} \frac{r|\dot{r}|}{\rho \tilde{R}_{\mathrm{a}}^{3}} \\
\approx & 10^{-11.5} \mathrm{~s}^{-1} \times \\
& \frac{r_{10}^{1 / 2} R_{*, 6}^{2} T_{*, 5}^{4}}{\rho \tilde{R}_{\mathrm{a}, \mathrm{km}}^{4}}\left(\frac{\bar{\Sigma}}{8.5 \mathrm{~g} \mathrm{~cm}^{-2}}\right)\left(\frac{\mid \dot{r}_{\mathrm{ss}, 1 \mathrm{AU} \mid}}{10^{-3} \mathrm{AU} \mathrm{Myr}^{-1}}\right)
\end{aligned}
$$

A rate $\dot{N}_{a} \sim 10^{-3}-1 \mathrm{~s}^{-1}$ is probably sufficient to account for nulling, so asteroids of $10 \mathrm{~m}$ size around NS slightly hotter than $10^{5} \mathrm{~K}$ or a disk more massive than $10^{-4} M_{\oplus}$ can bring the rate into this range.

The lifetime of the disk to inward migration is

$$
\begin{aligned}
\tau_{d} & \approx \frac{N_{a}}{\dot{N}_{a}}=\frac{r_{2}^{2}}{2 r|\dot{r}|} \\
& \approx 10^{3.1} \mathrm{Gyr} \frac{r_{2,-2}^{2} R_{\mathrm{a}, \mathrm{km}}}{R_{*, 6}^{2} T_{*, 5}^{4} r_{10}^{1 / 2}}\left(\frac{10^{-3} \mathrm{AU} \mathrm{Myr}^{-1}}{\dot{r}}\right)
\end{aligned}
$$

about ten times shorter than the collisional lifetime for nominal parameters. However, for parameter values that make the influx rate $\dot{N}_{\mathrm{a}}$ large, the disk lifetime will be substantially smaller, but still larger than the characteristic lifetime of a canonical radio pulsar.

\subsubsection{Collisional Migration}

A debris disk surrounding a neutron star has many characteristics similar to a planetary ring system and therefore many of the results of ring dynamics can be applied to this discussion. Inelastic collisions between particles determine the structure and evolution of a disk. On short time scales ( $\sim \tau^{-1}$ orbits, where $\tau$ is the optical depth of the disk) these collisions will result in the asteroids moving towards circular, coplanar orbits. On much longer timescales, these collisions widen the disk, with most of the mass transported inwards and most of the angular momentum transferred outwards.

Stewart et al. (1984) calculate the inward flux of material $\dot{m}$ in a viscous disk. This hydrodynamical result is consistent with a result from the more general approach in which the asteroid behavior is stastically modelled using the Boltzmann equation, presented in the same paper. They assumed that the asteroids are identical, indestructable, nonspinning spheres, which have a size much smaller than the inter-asteroidal spacing. Furthermore, they assume that the velocity dispersion of the particles has a Gaussian form, consistent with simulations of ring dynamics. The total flux $\dot{N}_{a}$ through radius $\mathrm{r}$ is then,

$$
\dot{N}_{a}=\frac{9}{2} \frac{1}{\rho R_{a}^{3}} \frac{1}{\Omega r} \frac{\partial}{\partial r}\left(r^{2} \Omega \Sigma \nu\right),
$$

where $\Omega$ is the Keplerian angular frequency and $\Sigma$ is the surface density. The viscosity $\nu$ was calculated numerically by
Goldreich \& Tremaine (1978), by balancing the energy dissipated during the inelastic collisions and the energy genertion due to the viscous stress.

$$
\nu \approx 0.15 \frac{v_{\mathrm{rms}}^{2}}{\Omega} \frac{\tau}{1+\tau^{2}} .
$$

Assuming that $\Sigma$, the velocity dispersion $v_{\text {rms }}$, and the optical depth $\tau$ do not vary greatly through the disk, the influx rate at the inner edge of the disk (set to be at the light cylinder) can be estimated:

$$
\dot{N}_{a}=10^{6.5} \mathrm{~s}^{-1} \frac{\tau}{1+\tau^{2}} r_{2,-2}^{-2} R_{\mathrm{a}, 2}^{-3} M_{\mathrm{a},-4} M_{*, 1.4}^{-\frac{1}{2}} \rho^{-1} v_{\mathrm{rms}}^{2} P^{\frac{3}{2}},
$$

with $P$ in seconds, $\rho$ in $\mathrm{g} \mathrm{cm}^{-3}$, and $v_{\mathrm{rms}}$ in $\mathrm{km} \mathrm{s}^{-1}$. Substituting in the previous expression for optical depth, and assuming it is much less than unity, we obtain

$$
\dot{N}_{a}=10^{5.3} \mathrm{~s}^{-1} r_{2,-2}^{-4} R_{\mathrm{a}, 2}^{-4} M_{\mathrm{a},-4}^{-2} M_{*, 1.4}^{-\frac{1}{2}} \rho^{-2} v_{\mathrm{rms}}^{2} P^{\frac{3}{2}},
$$

This migration rate is large and would provide material at a rate sufficient to disrupt emission processes, even if the mechanism for injecting material into the magnetosphere is inefficient.

\section{INSIDE THE MAGNETOSPHERE}

The standard picture is that coherent radio emission in pulsars requires counterstreaming particle flows that include $e^{ \pm}$ pairs produced in cascades. Sustained cascades require acceleration regions in the magnetosphere that yield particles energetic enough to radiate gamma-rays that then pair annihilate. As pulsars spin down, cascades cease as the electric fields in acceleration region(s) lessen. However, dormant regions can produce triggered pair avalanches that cause coherent radio transients.

\subsection{Asteroid Evaporation}

Asteroids rapidly evaporate when they reach radii below $10^{10} \mathrm{~cm}$ for $T_{*}=5 \times 10^{5} \mathrm{~K}$ and below $10^{9} \mathrm{~cm}$ for $T_{*}=10^{5} \mathrm{~K}$. Figure 5 shows evaporation times for objects of different size and evaporation fluxes assuming carbon composition (Cheng 1985). Figure 6 shows evaporation times vs. distance from the star, assuming that the asteroid surface is in equilibrium with surface radiation from blackbody radiation. These figures show that evaporation times are comparable to the orbital period only after asteroids are deep within the magnetospheres of long-period pulsars. Also, for larger objects $(\sim 100 \mathrm{~m})$, evaporation rates are comparable to the Goldreich-Julian particle rate, $\dot{N}_{\mathrm{GJ}}$, well before they are completely evaporated. These results confirm the basic picture that asteroid evaporation occurs at locations and with rates suitable for modification of magnetospheric electrodynamics. Moreover, the range of asteriod sizes expected for a highly collisional asteriod belt can account for the broad range of time scales seen in coherent radio emission. The curves shown in Fig. 6 do not include the effects of ohmic heating (mentioned earlier). Ohmic heating from $\mathbf{v} \times \mathbf{B}$ currents may be significant (see Appendix) during the final stages of evaporation and cause the evaporation times to vary more steeply with radius than shown in the figure. The relative importance of the various heating effects will be strongly object dependent, so we defer a detailed comparison to a future analysis. 


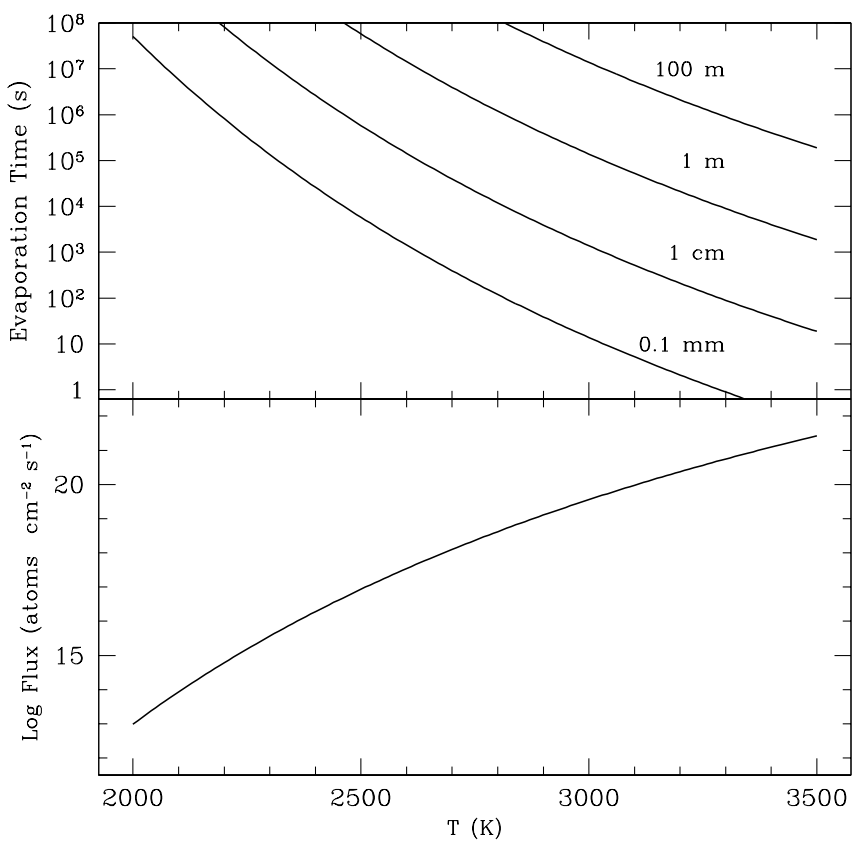

FIG. 5. - Evaporation of material from hot asteroids assuming an evaporation pressure for carbon using evaporation pressure data quoted from Chene (1985). Top: Evaporation times for different sizes. Bottom: Evaporation flux.

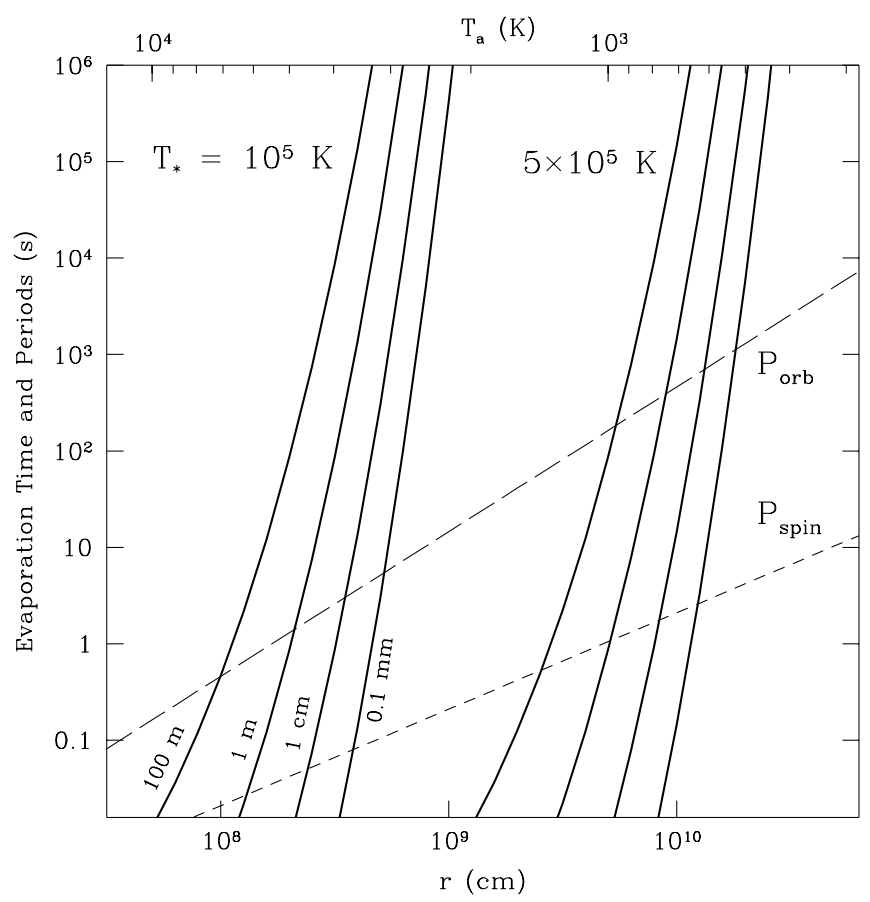

FIG. 6.- Evaporation times for asteroids of different sizes for two different values of NS surface temperature, as labelled. Also shown (dashed lines) are the Keplerian orbital period and the spin period, the latter assuming that $r$ corresponds to the light cylinder radius.

\subsection{Electromagnetic Capture}

Ionized evaporant from an inspiraling or infalling asteroid is rapidly spun up to the NS's spin rate, $\Omega_{*}$ owing to the typ- ically small electron gyro-radius, $r_{g} \approx 10^{2.3} \mathrm{~cm}\left(r / R_{\mathrm{LC}}\right)^{4} P^{3}$ (without relativistic corrections and ignoring asteroidal motion). The detailed trajectory of the material depends on its field-parallel velocity prior to ionization (e.g. collisional motion) and on any parallel electric fields. Because the lifetime for many asteroids exceeds a spin period, an asteriod and its evaporant will encounter gaps. Ionized material is accelerated inside such gaps, contributing to the current flow in the magnetosphere, some of which is subsequently lost to flows outside the LC. The fate of material captured in the closed magnetosphere is not clear. It can remain tied to magnetic field lines but some may diffuse to larger radii or the open field-line region from which it is accelerated to the NS or lost by the magnetosphere.

\subsection{Currents and Gaps}

Pulsar magnetospheres will adjust the charge density to $n_{\mathrm{GJ}}$ in order to become force free (i.e. vanishing electric field parallel to $B, E_{\|}=0$ ). But boundary conditions (or, as formerly assumed, large work functions at the NS surface) prevent this in "gaps" where $E_{\|} \neq 0$. Gap sites include the polar cap above the NS surface in the open field line region (Ruderman \& Sutherland 1975), the slot gap at the interface between the open and closed field-line regions (e.g. Scharlemann et al. 1978; Arons 1983; Muslimov \& Harding 2003) and the outer gap that bounds the null surface $\Omega$. $B(r)=0$ (e.g. Cheng et al. 1986; Romani \& Yadigaroglu 1995; Cheng et al. 2000; Hirotani et al. 2003; Zhang et al. 2004; Takata et al. 2006).

Particle acceleration in gaps can yield copious gamma-ray emission that drives electron-positron $\left(e^{ \pm}\right)$pair cascades if there is sufficient residual field $E_{\|} \neq 0$, which will depend on other sources of charged particles, such as thermionic emission from the NS surface or injection of charges from external sources.

We assume that the standard picture is at least approximately correct because (a) acceleration of particles to high energies in the open field-line region is clearly needed to produce beamed radio emission that is consistent with the observed widths and polarization of pulses; (b) subpulse drift behavior is qualitatively consistent with $\mathbf{E} \times \mathbf{B}$ drifts around the magnetic axis, which rely on the existence a gap field; and (c) if surface magnetic field estimates are correct, the conditions exist for pair production to occur. Jessner et al. (2001) discuss an alternative to this view. Whether pair production is in the form of sustained cascades or in episodic avalanches is open to question for particular objects, with the answer dependent on NS properties and on external triggers. Since the accelerating field $E_{\|} \propto \rho_{\mathrm{e}}(r)-\rho_{\mathrm{e}, \mathrm{GJ}}(r)$ in the corotating frame, any excess particles injected from outside the LC that diminish the charge density difference (or change its sign) must drastically alter the population of particles and the fraction of $n_{\mathrm{GJ}}$ needed to do so can be small. Drifting subpulse patterns are sometimes coherent, other times disrupted, suggesting that the $\mathbf{E} \times \mathbf{B}$ drift rate is time variable. Our model provides a natural mechanism for this variability.

\subsection{Coherent Radio Emission}

In our model, neutral particles enter the magnetosphere as rocks or grains, dissociate and ionize from thermal and perhaps magnetospheric radiation, and perturb the current flow. In a pulsar with an active cascade, the intruding charges can reduce $E_{\|}$and thus the density of cascade $e^{ \pm}$pairs. Presumably this reduces the altitude of radio emission, which is tied 
to one of the plasma frequencies defined in the system, or completely detunes the coherent amplification by altering the growth rate of the plasma instability that provides the coherence. As NS spin down, cascades cannot be sustained and gap accelerators become quiescent. Charges from accreted rocks may temporarily re-activate the $e^{ \pm}$cascade in one or more of the gaps, leading to a burst of coherent radio emission.

Enough uncertainty exists about the structure of these gaps that we sidestep any detailed modeling of the process by which deconstructed asteroids disrupt the electrodynamics. Disruption undoubtedly depends on NS properties such as the surface temperature and spin rate. It also depends on the trajectories of incoming asteroids, whether they slowly spiral in to regions where $E_{\|} \neq 0$, or alternatively, are on highly eccentric orbits arising from collisions or resonances outside the magnetosphere.

There appear to be ample configurations and mechanisms by which disruption can occur, including (a) direct injection of charges into the polar-cap gap and slot gap; (b) perturbation of the outer gap by itself; and (c) direct injection into the outer gap, which triggers an inward moving cascade that perturbs the inner gap region(s). We consider the most likely case to involve contamination of the outer gap, primarily because it borders the LC and its volume presents a large target for incoming material (e.g. Takata et al. 2004, 2006). In long-period, cool pulsars, the outer gap is unable to sustain pair cascades that involve pair production from gap gammarays interacting with X-rays from the NS surface. However, an external flux of particles can trigger an avalanche that, in turn, may perturb the inner-gap region of the magnetosphere. Such perturbation could enhance the pair production in some cases or it could quench the pair production in others. Consequently, perturbation of the outer gap may lead to a burst or a cessation (i.e. a null) of radio emission.

Perturbation of a gap region by injected charges may increase pair production in some objects and decrease it in others, depending on whether the region is "starved" of particles and on the potential drop across it. In active radio pulsars with a steady $e^{ \pm}$cascade sustained by a slight deficit from the GJ charge density, injected charges will diminish $E_{\|}$, thus reducing the energies of accelerated particles and the number of radiated gamma rays that can pair produce. The pulsar remains radio loud if the conditions are sustained for driving a plasma instability that is responsible for the coherence and if coherence occurs at detectable radio frequencies. A change in radio emission altitude is expected and it could be lowered if it depends solely on plasma densities or it might increase if the instability growth rate becomes longer. In objects that are mostly radio-inactive because they cannot sustain pair cascades, injected charges can provide the seed particles needed to initiate a short-lived cascade.

External particles need not provide the full GJ current in order to perturb the radio emission, so the actual mass needed to produce observable effects is probably orders of magnitude smaller than given in $\$ 3.2$ In fact, if the GJ charge density were variable by $100 \%$, large torque changes would be produced. Depending on the time scales of such variability, torque fluctuations would be manifested as an observable contribution to the ubiquitous timing noise found in pulsars.

\subsection{Emission Altitude and Beaming}

Most pulsar models invoke the two-stream instability as the source of plasma bunching that underlies the strong coherence of the radio emission (e.g. Ruderman \& Sutherland
1975; Usov 1987; Asseo \& Melikidze 1998). Models differ in the nature of the two streams but assume that the instability's growth time must be smaller than the propagation time from the NS surface, from which the plasma originates and propagates at $c$. This requirement is highly restrictive and leads to the proposal (Usov 1987; Asseo \& Melikidze 1998) that variability near the NS surface causes particle clouds to overtake those from earlier discharges and leads to suitable instability growth times. When particles are injected into the magnetosphere from asteriods, they provide an additional stream of particles that can enlargen the parameter space for producing coherent radio emission.

In the two-stream instability, the maximum growth rate is at a frequency proportional to the local plasma frequency, which is a function of radius, $\omega_{p} \propto\left(n_{ \pm} / \gamma\right)^{1 / 2}$, and which also depends on relevant Lorentz factors for the particle streams. The equation for a dipolar field line, $r(\theta)=R \sin ^{2} \theta / \sin ^{2} \theta_{0}$, where $\theta_{0}$ is the angle of a field line at the NS surface, implies that emission from relativistic particles is beamed in directions parallel to the field line tangents that make an angle $\theta / 2$ from the radial vector. Emission is therefore beamed into polar angles $\theta_{e}=3 \theta / 2$, for small angles near the magnetic axis and well inside the LC. If the Lorentz factor remains constant, a change in pair density induces corresponding changes in emission altitudes and angles,

$$
\frac{\delta n_{ \pm}}{n_{ \pm}}=-3 \frac{\delta r}{r}=-6 \frac{\delta \theta_{e}}{\theta_{e}}
$$

where the minus signs signify that an increase in pair density induces a reduction in emission altitude and emission angle. Asteroidal injection may enhance or diminish the pair density, so both increases and decreases can be expected. A given object may show a change in pulse profile (mode change), become too weak to detect because its beam narrows and thus misses the line of sight, or become detectable as a result of a wider beam.

\subsection{Subpulse Drift Rates}

Modulation of the charge density by injected charges alters not only $E_{\|}$but also the $\mathbf{E} \times \mathbf{B}$ drift of structures within a gap region, such as those offered as explanations for the drifting subpulse phenomenon. Ruderman \& Sutherland (1975) derive a drift velocity for the polar-cap gap region

$$
v_{d} \approx \frac{c \Delta \Phi}{B_{s} R_{*}^{3 / 2}}\left(\frac{c P}{2 \pi}\right)^{1 / 2} \approx 10^{3.8} \mathrm{~cm} \mathrm{~s}^{-1} P^{1 / 2} B_{12}^{-1} \Delta \Phi_{12},
$$

where $\Delta \Phi=10^{12} \Delta \Phi_{12}$ volts is the typical potential drop along the field lines in the gap. Injected charges will alter this potential drop, so the drift velocity will be affected proportionally.

\subsection{Spindown Energy Loss}

Cheng (1985) writes the total spindown luminosity as

$$
\dot{E}_{\text {total }}=\dot{E}_{\mathrm{rad}}+\frac{\Omega^{4} B^{2} R^{6}}{c^{3}}\left(\frac{I_{*}}{I_{\mathrm{GJ}}}\right),
$$

where the first term is the contribution from low-frequency dipole radiation and the second term results from the torque exerted by magnetospheric currents; $I_{*}$ is the actual current and $I_{\mathrm{GJ}}$ is the Goldreich-Julian current. Cheng considered interstellar grains that intrude into the LC and contribute to $I_{*}$. 
For material originating from a circumpulsar disk, the effects are largely the same, but the delivery of extrinsic material and its likely episodic behavior are different. Intrinsic contributions to $I_{*}$ include ions and $e^{ \pm}$pairs. Without an extrinsic component, the current may be steady with shot-pulse modulations on the light-travel time across accelerating gaps $(\ll P)$ or (Cheng \& Ruderman 1980; Jones 1982) it may show cyclical behavior on thermal time scales for the polar cap, which Jones (1982) estimates to be several thousand seconds. By introducing an extrinsic contribution that has a wide range of time scales, the rich variety of radio intensity variations and their variation from object to object - can be explained.

\subsection{Torque Fluctuations and Timing Noise}

As discussed earlier, TOA variations will result from the recoil of the NS in response to an ensemble of orbiting asteriods. Accreting asteroids will induce true spin fluctuations of the NS through two mechanisms. First, spinup of a mass $m_{a}$ through electrical capture in the magnetosphere implies corresponding spindown of the NS. Second, an increase in current from injected charges will produce a torque pulse that acts on the NS. We consider these in turn.

Suppose that an effective fraction $f_{m}$ of the mass $m_{a}$ of an asteroid is ultimately spun up into corotation from an orbit that is much slower than the NS spin. Once ionized, ions and electrons will be accelerated in opposite directions, so the spunup mass will be significant only for cases where ions are accelerated inward. When ions are accelerated outward and lost from the magnetosphere, a torque will be applied to the NS if the net acceleration is non-radial, as is likely. Thus, we expect in either case that the effective value of $f_{m}$ may be sizable. The change in NS spin rate for an electrical capture radius, $r_{c}$, is

$$
\Delta \Omega_{*}=-\left(\Omega_{*} m_{a} r_{c}^{2} / I_{*}\right)\left(1-\Omega_{K} / \Omega_{*}\right)
$$

where $I_{*}$ is the NS's moment of inertia and $\Omega_{K}$ is the Keplerian angular velocity, which we assume is much less than $\Omega_{*}$ because objects evaporate well outside the corotation radius. The resulting random walk in spin rate will have a strength parameter (e.g. Cordes \& Greenstein 1981)

$$
\begin{aligned}
S_{\mathrm{FN}} & =\mathcal{R}\left(\Delta \Omega_{*} / 2 \pi\right)^{2}=\mathcal{R}\left(f_{m} m_{a} r_{c}^{2} / I_{*}\right)^{2}\left(\Omega_{*} / 2 \pi\right)^{2} \\
& =10^{-25.3} \mathrm{~s}^{-3} \frac{\mathcal{R}}{10 \mathrm{yr}^{-1}}\left(f_{m} \rho R_{\mathrm{a}, \mathrm{km}}^{3} r_{c, 10}^{2} / I_{45} P^{2}\right)^{2},
\end{aligned}
$$

where $\mathcal{R}$ is the event rate, assumed equal to the asteroid injection rate, and parameter values were chosen to yield strength parameters and rms pulse phases similar to those inferred from pulsars (e.g. Cordes \& Helfand 1980; Cordes \& Downs 1985; Arzoumanian et al. 1994; Hobbs et al. 2004). The rms pulse phase variation over a time span $T$ is

$$
\begin{aligned}
\sigma_{\phi}(T)= & \left(\frac{S_{\mathrm{FN}} T^{3}}{12}\right)^{1 / 2} \\
\approx & 10^{-1.9} \mathrm{cy} \times \\
& \left(\frac{\mathcal{R}}{10 \mathrm{yr}^{-1}}\right)^{1 / 2}\left(\frac{f_{m} \rho R_{\mathrm{a}, \mathrm{km}}^{3} r_{c, 10}^{2} T_{\mathrm{yr}}^{3 / 2}}{I_{45} P^{2}}\right),
\end{aligned}
$$

which is comparable to measured rms timing noise variations for nominal parameter values if the effective fraction $f_{m}$ is not small.

The second mechanism, fluctuations in current, will alter the torque on a NS to produce spin noise.
Cordes \& Greenstein (1981) considered a superposition of torque pulses associated with nulling and modeled the resulting timing noise, which also would be a random walk in the spin frequency. Episodic accretion events that produce torque pulses of duration $W$ by a fractional change in current, $f_{I}=\delta I_{*} / I_{*}$, yield steps in spin frequency, $\Delta \Omega_{*}=f_{I} \dot{\Omega} W$. The strength parameter for the random walk, $S_{\mathrm{FN}}=\mathcal{R} W^{2}\left(f_{I} \dot{\Omega} / 2 \pi\right)^{2}$, corresponds to an rms spin-phase (for $\left.W=10^{3} W_{3} \mathrm{~s}\right)$

$$
\sigma_{\phi}(T) \approx 10^{-2.8} \text { cy } f_{I}(\mathcal{R} W)^{1 / 2} W_{3}^{1 / 2} \dot{P}_{-15} P^{-2} T_{\mathrm{yr}}^{3 / 2},
$$

also comparable to measured timing noise. Asteroidal intrusion into the LC is more likely in older, long-period pulsars with small values of $\dot{P}$ for which $\sigma_{\phi}$ is small, whereas measured timing noise is larger for short-period objects with large $\dot{P}$. It seems likely that there are several processes that contribute to timing noise, with extrinsic current sources playing a major role only in some objects. The recent discovery of $50 \%$ increases in $\dot{P}$ in pulsar B1931 +24 (Kramer et al. 2006) with $W \approx 10 \mathrm{~d}, \mathcal{R} W \sim 0.2$, and $f_{I} \sim 1$ is consistent with this picture for timing noise, albeit for a case where the individual torque events are easily seen in the timing data. For pulsars with faster rates and shorter event durations, $W$, the torque events may be inferred only statistically rather than through individual changes in $\dot{P}$.

The ratio of spin-frequency perturbations for the two cases is

$$
\frac{\Omega_{*}^{(2)}}{\Omega_{*}^{(1)}}=\frac{f_{I} W I_{*} \dot{P}}{f_{m} m_{a} r_{c}^{2} P} \approx 10^{-2} \frac{f_{I} W_{3} I_{45} \dot{P}_{-15}}{f_{m} m_{a, 15} r_{c, 10}^{2} P} .
$$

Spindown from mass loading dominates for the rather large effective asteriod masses assumed $\left(f_{m} m_{a}=10^{15} f_{m} m_{a, 15} \mathrm{~g}\right)$, and the large capture radius; adjustment of these and other parameters can modify the ratio by several orders of magnitude either larger or smaller.

Asteroid-induced torques may also induce changes in spinaxis orientation, which would amplify their effects on the measured pulse phase. The change in orientation will depend on how the instantaneous current density deviates from the average current density, so is hard to predict. If the mean torque depends on the angle $\alpha$ between the spin and magnetic axes as $f_{\alpha}$, then the induced change in $\dot{P}$ will be (Cordes 1993) $\delta \dot{P} \sim \delta \theta \dot{P}\left(\partial f_{\alpha} / \partial \alpha\right)$. Even very small angular changes can produce easily detectable, accrued pulse-phase changes from this effect.

\section{APPLICATIONS}

\subsection{Nulls, Drifts and Mode Changes}

Charges injected from asteroids may be sufficent in number that they reduce or shut off the pair cascade and thus alter or extinguish the radio emission. Several cases may apply. First, injected charges may directly perturb the flow in the gap region that is responsible for producing the radio-emitting particle flow; it is commonly assumed that this is the polarcap gap at the NS surface. In this case, we would also expect the current to increase during a null, leading to an increase in spindown torque, as described above. Alternatively, asteroid charges are injected into an outer-gap region, producing an avalanche of pair production with one charge sign flowing into the inner gap.

All things considered, we expect nulls to be most prominent in cool, long-period pulsars for which a wide range of asteroidal conditions can yield influx into their large LCs. Nulls 
are not impossible in younger, hotter objects, but the requirements are more selective on asteroid size and on trajectories into the LC. From a given orbital radius, the LC is a fraction $\sim(c P / 4 \pi r)^{2} \ll 1$ of the sphere, so collision fragments large enough to avoid evaporation outside the LC must be on highly restricted trajectories to hit the target.

Pulsars that show nulling often also show drifting subpulses with variable drift rates and changes in pulse profile (mode changes). In some cases, drift rates jump between two or more preferred values. We interpret such objects as ones that can sustain current flows and pair-production cascades, on average, but that are modulated by accreted asteroids according to the processes we have identified. Injected charges will alter the local difference between the actual and Goldreich-Julian charge and current densities, $\rho-\rho_{\mathrm{GJ}}$, which will change the parallel electric field, $E_{\|}$. Boundary conditions at the interfaces between the gap, the closed-field-line region, and the NS surface then imply changes in the transverse electric field and, hence, in the $\mathbf{E} \times \mathbf{B}$ drift rate. Injected charges therefore can account for variations in drift rate and it is not surprising to find objects that show both nulls and variable drift rates. Why should drift rates have preferred values? This is equivalent to asking, why should there be quantized values of the parallel electric field? We offer no definitive answer, but it seems possible that particle source regions in the polar-cap region, which drift and themselves appear finite in number (e.g. Deshpande \& Rankin 1999), may appear and disappear as discrete entities. Alternatively, if the current and particle flows consist of several particle types, with one or more depending on injection from external sources, the current and potential drop may show discrete states. Finally (Gogoberidze et al. 2005) propose that subpulse drift is associated with drift waves in the open field-line region of the magnetosphere that modulate the particle accleration. Asteroidal influx, in turn, would modulate this modulation.

\subsection{A Cue-ball Model for Quasi-periodic Bursts and Nulls in B1931+24}

The long 40 d quasi-periodic bursts from B1931+24 with about $20 \%$ duty cycle represent a time scale that is very hard to understand from processes internal to the magnetosphere or the NS. Periods of about this duration are associated with Tkachenko oscillations of superfluid vortices (Coddington et al. 2003), but presumably these are cyclical with high oscillation " $Q$ " from which it is not clear how lowduty cycle bursts would be produced. Orbital processes seem richer in possibilities and thus more promising.

B1931+24 itself is a typical canonical pulsar $(P=0.81$, average $\langle\dot{P}\rangle=10^{-14.1}$, and spindown age $\left.\tau_{s}=P / 2\langle\dot{P}\rangle=1.6 \mathrm{Myr}\right)$ yet the effects seen from it (bursts and nulls, a 50\% increase in $\dot{P}$ during bursts, and no short nulls during a burst) are uncommon among known objects. Observational incompleteness due to the impracticality of continuous sampling of large regions of the sky may play a role, but the observed effects are probably physically rare. Therefore we seek special circumstances to explain the observed phenomena.

\subsubsection{Eccentric Asteroid Model}

In the context of our model, the burst period and duty cycle are naturally explained with a large asteroid of radius $R_{b}$ moving in an eccentric 40-d orbit with semi-major axis $a=0.26 \mathrm{AU}\left(P_{\mathrm{orb}} / 40 \mathrm{~d}\right)^{2 / 3}$. At this distance, $R_{b}$ can be as large as $100 \mathrm{~km}$ (c.f. Figure 4). This "cue-ball" or "mini-
Nemesis" asteroid ${ }^{3}$ induces observable effects by deflecting smaller asteroids into the loss-cone for light-cylinder insertion in much the same way that solar-passing stars perturb comets in the Oort cloud. Injection into the magnetosphere would be stochastic, so the observed continuity of the bursts, once they commenced, suggests efficient injection of a large number of objects. Burst durations also would be stochastic. We consider implausible a model where the cue-ball asteroid directly injects charges into the magnetosphere. To do so requires an eccentricity very near unity in order that the orbit's minimum radius $a(1-e)$ be comparable to the LC radius. Tidal heating (c.f. Eq. 20) is also unlikely to be significant for an asteroid with $40 \mathrm{~d}$ orbital period with reasonable eccentricity.

During short duty cycle bursts, the spindown rate increases, suggesting that the asteroid induces enhancements to the current flow. We associate such episodes to the periastron of a large asteroid because, in an elliptical orbit, an object spends less time nearer than farther from the star. For example in an orbit with $e=0.61$, the cue-ball asteroid spends $20 \%$ of its time at radii less than twice the minimum NS distance of $a(1-e)$. If only a $10 \%$ increase in distance is sufficient to terminate the bursts, an eccentricity of 0.25 is implied. The opposite case, where bursts are associated with apastron and a termination of asteroidal influx, would imply a lowereccentricity orbit but does not readily explain the increase in $\dot{P}$ during bursts.

For a large asteriod in the densest part of the disk of small asteroids, the mean time between collisions is (c.f. Eq. 30.

$$
t_{c}=10^{7.4} \mathrm{~s} \frac{\epsilon_{h} \rho}{\Delta v}\left(\frac{R_{a}}{R_{b}}\right)^{2} R_{\mathrm{a}, 2} r_{2,-2}^{3} M_{a,-4}^{-1},
$$

where $\Delta v$ is the relative velocity. This velocity difference is, for small asteroids in circular orbits of size equal to the periastron distance of the cue-ball asteriod, $\Delta v=$ $\sqrt{\left.(1+e)^{1 / 2}-1\right)(G M / r)} \sim(e / 2) v$, a potentially large velocity. For nominal parameters (Eq. 26 32) $t_{c} \sim 1 \mathrm{~s}$ for $R_{b} / R_{a}=10^{3}$ (i.e. $R_{b}=1 \mathrm{~km}$ ), implying a large number of collisions per orbit, which may imply that the cue-ball asteriod's orbit will evolve too quickly. Thus, either the periastron of the cue-ball asteriod is outside the densest part of the asteroid disk or the disk is much less dense than our nominal disk.

The eccentricity of a cue-ball asteriod suggests that the object is part of a family of large objects that co-exists outside the small asteriod belt of objects that are injected into the pulsar's magnetosphere. This follows from the need to account for the cue-ball's eccentricity. In the solar system, near-Earth objects (NEOs) exist as a result of chaotic dynamics in mainbelt asteroids induced by resonances with Jupiter and possibly Mars (Murrray \& Dermott 1999). Thus there may be additional large objects orbiting B1931+24. Given the large change in $\dot{P}$ in the on state for the pulsar, it is possible that orbital recoil effects on the TOAs of the pulsar are masked. Thus it is of interest to carefully analyze timing data to search for the individual effects of multiple orbiting objects.

\subsection{Orbital and Disk Lifetimes}

Requirements on the orbital collision model are that (a) the cue-ball orbit must be stable for a long period of time, $t_{b}$, that

\footnotetext{
${ }^{3}$ Nemesis is the name of the putative solar companion star that was hypothesized to be the cause for quasi-periodic, terrestrial extinction events with period $\sim 36$ Myr due to perturbation of the Oort cloud of comets (Raup 1986).
} 
we take to be $t_{b}=10^{5} t_{\mathrm{b}, 5} \mathrm{yr}$. and (b) the influx into the LC of the pulsar must be at a rate that can yield continuous and sustained bursting over a $\sim 8$-day period. The first condition places a constraint on the change in orbital momentum per orbital period based on collisions occuring at a rate $t_{c}^{-1}$ for a fraction of the orbital period, $f_{b} P_{\text {orb b }}$. This yields

$$
\frac{\Delta p_{b}}{p_{b}}=\left(\frac{m_{a}}{m_{b}}\right)\left[\frac{\Delta v(r)}{v_{b}(r)}\right]\left(\frac{f_{b} P_{\text {orb }, \mathrm{b}}}{t_{c}}\right) \lesssim \frac{P_{\text {orb }, \mathrm{b}}}{t_{b}} .
$$

The second condition requires that the collisional influx into the LC must be at a high-enough rate that the magnetosphere receives a continuous flow of evaporated material during a burst. Assuming collision fragments are directed isotropically, the fraction that enters the LC is $f_{\mathrm{LC}} \approx(c P / 4 \pi r)^{2}$, yielding a mean time between injections of $t_{c} / f_{\mathrm{LC}}$ that we require to be less than the evaporation time of an asteroid, $t_{\text {evap. }}$. Taken together and using $\Delta v(r) / v_{b}(r) \sim e / 2$, we can bracket the collision time using

$$
\frac{e f_{b} t_{b}}{2}\left(\frac{m_{a}}{m_{b}}\right) \leq t_{c} \leq f_{\mathrm{LC}} t_{\mathrm{evap}}
$$

Evaluating for $m_{a} / m_{b}=\left(R_{a} / R_{b}\right)^{3} \approx 10^{-15}$ (e.g. for a $100 \mathrm{~km}$ cue-ball and $\left.R_{a}=1 \mathrm{~m}\right)$, assuming that collisions take place at periastron, $r=a(1-e)$, and using an evaporation time $t_{\text {evap }}=$ $10^{4} t_{\text {evap }, 4} \mathrm{~s}$ (which applies to meter-size rocks for a variety of temperatures), we obtain

$$
10^{-4.1} \mathrm{~s} f_{\mathrm{b}, 5}\left(\frac{m_{b} / m_{a}}{10^{15}}\right) \leq t_{c} \leq 10^{-2.4} \mathrm{~s} t_{\mathrm{evap}, 4} .
$$

This demonstrates that the orbital configuation can be maintained for a sufficiently long time that we do not require ourselves to be observing at a special time.

\subsection{Other possibilities}

Analogs to other solar-system phenomena may apply, though they do not readily yield a quasi-periodicity in the magnetospheric response. For example, "horseshoe" orbits of small asteroids that enclose the $L_{3}-L_{5}$ Lagrangian points in a NS-large-asteroid system have libration periods that can be large multiples of the orbital period. These orbits span a range of distances from the NS that is wider at the $L_{4,5}$ points than at the $L_{3}$ point. For orbits that are closer to the NS, one might expect an increase in collisions or evaporation rate that could enhance injection into the LC. Only if the orbits are populated with a small number of objects would we expect to see episodic or quasi-periodic effects, however.

As another variant, the $40 \mathrm{~d}$ quasi-period may be associated with the period of interaction between two orbiting objects in resonance. Mean-motion resonances, such as those that produce gaps within the solar system's asteroid belt and in planetary ring systems, induce chaotic motions that could drive asteriods into the NS. However, this process is likely to produce a more continuous and possibly large collision rate and, hence, less-intermittent bursts. Consequently, we favor the cue-ball model for producing the quasi-periodic effects in B1930+24.

\subsection{Rotating Radio Transients (RRATs)}

RRAT objects identified to date populate the same part of the $P-\dot{P}$ diagram as objects that show prominent nulling, with the possible exception of J1819-1458, which has a large spindown-derived magnetic field that is similar to that of magnetars. We consider RRATs to be associated with NS that are similar or identical to canonical pulsars. They are observed to be quiescent most of the time either because they are intrinsically so or because their radio beams do not typically point toward us. RRAT pulses have duty cycles that are significantly smaller than those of canonical pulsars McLaughlin et al. (2006) with comparable periods, suggesting that the intermittent emission is from a region of the magnetosphere smaller than typical.

If RRAT objects are active pulsars not usually beamed toward us, their intermittency could be explained by the injection asteroidal material into the outer magnetosphere. The pair production rate would then be enhanced, which would increase the overall plasma density and the altitude of emission. As discussed earlier, this would increase the probability of detection and make some objects intermittently detectable. Pulses from such objects would be wider than typical if the entire open field line region is affected. Only if a restricted region were affected might we expect to see narrow pulses. This appears to be the case with canonical pulsars for which single pulses are almost always narrower than the average of many pulses, signifying that the open field line region is not instantaneously filled with coherent emitters.

A perhaps more reasonable alternative is that asteroidal material triggers an inactive outer gap. It is easier to inject material into an outer gap and such gaps should exist in all pulsars. In long period pulsars, however, outer gaps have potential drops too small to sustain self-generated pair cascades (Zhang et al. 2004). However, external triggers can drive pair avalanches in such gaps. It is noteworthy that for objects where outer-gap emission is a plausible candidate source of radio emission, the radio pulse components are quite narrow. The best cases are the Crab pulsar and the MSP B1937+21, both of which show high-energy emission that aligns in pulse phase (or nearly so) with the radio components. These two objects show giant pulses in these same components. Giant pulses from the Crab pulsar are very narrow if one corrects for pulse broadening from radio-wave scattering, e.g. $W / P \sim 100 \mu \mathrm{s} / 33 \mathrm{~ms} \sim 0.3 \%$. The pulses from B1937+21 Jenet. Anderson \& Prince (2001) are a wider $\sim 2.5 \%$. By triggering the outer gap, pair production and coherent emission may be produced directly and we may see the emission from either the ingoing or outgoing beam. In this regard, our model is similar to that of Zhang. Gil \& Dyks (2006), who postulate intermittency and reversal of beaming direction but did not propose a specific mechanism for the intermittency, as we do here. Alternatively (or in addition), activation of the outer gap may induce particle flows that in turn activate or alter processes lower in the magnetosphere, such as the polar cap.

Our model implies that a triggered avalance would continue for only a finite amount of time. However, it can be longer than a NS spin period if accretion is sustained.

In some of the RRATS we may expect to see polar cap emission from the pulsar. Thus, a test of the model would be to search for this emission. We would expect to see these components at quite different pulse phases.

\section{TESTS AND FURTHER APPLICATIONS}

We have suggested a number of plausbility arguments in favor of our model, including the distribution of nulling and RRAT pulsars in the $P-\dot{P}$ diagram (Figure 1), the temperature and spin rate regime in which we expect disk material can enter a pulsar's magnetosphere in a largely neutral state (Figure 4), and the distribution of spin-magnetic-moment an- 
gles in Figure 2 Here we consider ways in which the model can be tested and used to infer the properties of material orbiting radio pulsars.

As possible tests, we suggest that identification of counterexamples to the trends seen in the $P-\dot{P}$ diagram would be valuable. For example, identifying nulls in MSPs or in young pulsars with high thermal luminosity or high magnetospheric luminosity combined with favorable orientation would suggest alternative causes in those objects. In the case of B1931+24, the pulsar showing quasi-periodic bursts with correlated increase in $\dot{P}$, we suggest that there may be multiple orbiting objects that may be detectable as arrival time perturbations that must be disentangled from the changes in $\dot{P}$. A careful investigation of the "timing noise" in a large sample of pulsars, such as that presented in Hobbs et al. (2004), may reveal trends in types of timing noise vs. $P$ and $\dot{P}$ and may corroborate the existence of torque fluctuations in those objects that also show nulls and other evidence for accretion.

\subsection{Young Pulsars}

Injection of charged particles through asteroid intrusion is much less likely from young pulsars that have small magnetospheres (if they have low spin periods), have hot surface temperatures $\left(\gtrsim 10^{6} \mathrm{~K}\right)$, and have high non-thermal luminosities. Exceptions may exist if occasional, large asteroids on low-angular-momentum trajectories can rapidly get inside the LC before they are evaporated. Thus, the division between "young and hot" and "old and cool" NS may depend on the collision rate and size of the asteroid disk, which will determine the rate at which events might be seen from a given object. Already, the trend appears to be that objects near the death line in the $P-\dot{P}$ diagram are much more prone to showing transient effects that can be attributed to asteroids. Nonetheless, we may expect to see occasional departures from this trend. A notable case may be RRAT J1819-1458, which has a small spindown age (117 kyr) but has a long period (4.3 s) (McLaughlin et al. 2006). Thus its LC is large but its surface temperature (Revnolds et al. 2006) is also large, $T_{*} \approx 0.12 \mathrm{keV} \approx 10^{6.1} \mathrm{~K}$. Larger asteroids $(\gtrsim 100 \mathrm{~m})$ can enter, though not deeply, into the magnetosphere before they evaporate, allowing the possiblity that they trigger a dormant outer-gap region.

\subsection{Millisecond Pulsars}

Most MSPs have white-dwarf companions in compact orbits, so those objects are unlikely to have additional debris given their large ages $(0.1-10 \mathrm{Gyr})$. Disks may exist around isolated MSPs as condensed evaporant from the companion star that once provided accreting material responsible for spinup of the MSP. Such disks may disappear on time scales much shorter than MSP radio-emitting lifetimes. For cases where there are disks, MSPs are unlikely to allow injection of asteroids into their magnetospheres because their light cylinders are very small, $r_{\mathrm{LC}}=10^{7.4} \mathrm{~cm}(P / 5 \mathrm{~ms})$, and the nonthermal radiation flux typically is high.

\subsection{Infrared Disk Detection}

Unlike the recent detection of a fairly massive disk around the magnetar 4U 0142+61 (Wang. Chakrabarty \& Kaplan 2006), disks around canonical radio pulsars will be much more difficult to detect.

Bryden et. al. (2006) recently observed pulsar planetary system B1257+12 in the mid-infrared looking for asteroidal dust. No evidence was found for emission, but the limits do not rule out a disk comparable to the Solar System's asteroid belt. We expect the planetary system around B1257+12 is less likely to contain a massive asteroid belt, because gravitational interactions will remove debris at a much faster rate than for an asteroid belt without planets. For the objects we consider, the disk is much more compact about the central source, so the emission should peak in the near infrared.

We assume that the disk, with mass $M_{a}$, is composed of spherical asteroids of area $r_{a}$, density $\rho$, and albedo A. The disk is heated by the thermal radiation of the neutron star (radius $R_{*}$, surface temperature $T_{*}$ ). We also assume the disk is orthogonal to our line of sight. The temperature $T_{a}$ of the asteroid at distance $r$ from the NS is given by Eq. 18 As the disk is very small, an observer will see the disk as a point source. To calculate the total flux density, we add the flux directed towards us from all the asteroids:

$$
F_{\nu}=\int_{r_{1}}^{r_{2}} d r \pi r_{a}^{2} F_{\nu, \mathrm{em}}\left(T_{a}\right) \frac{d N}{d r}
$$

where $d N / d r=2 \pi r n_{a} \epsilon_{h} r_{2}$ is the number vs. $r$ and we assume uniform number density, $n_{a}$. Adding up the Planck flux from each asteroid, we obtain

$$
F_{\nu}=\frac{45}{8 \pi^{5} d^{2}} \frac{\sigma_{\mathrm{SB}}(1-A) T_{*}^{4}}{\nu} \frac{R_{*}^{2}}{r_{a} r_{2}^{2}} \frac{M_{a}}{\rho} \int_{x_{1}}^{x_{2}} \frac{d x x^{3}}{e^{x}-1},
$$

where $x_{1,2}=\left(h \nu / k T_{*}\right) \sqrt{2 r_{1,2} / R_{*}}$. Using our standard disk parameters (Eq. 26 32), the peak flux occurs at a wavelength $\lambda \approx 13 \mu \mathrm{m} T_{5}^{-1} R_{6}^{\frac{1}{2}}\left(r_{2,-2} r_{1,10}\right)^{\frac{1}{4}}$, when the peak of the integrand $(x \approx 2.8)$ occurs at the geometric average of the two endpoints. The integral is approximately 3 for the standard parameters but could be as high as $\approx 6$ for asymptotically large disks, and will be much smaller for narrower disks. However, narrower disks would not satisfy the contraints suggested in $\$ 3.2$ We find then that the observed flux density is

$$
F_{\nu}=10^{-1.4} \mu \mathrm{Jy} d_{\mathrm{kpc}}^{-2} T_{5}^{3} R_{6}^{\frac{3}{2}} M_{\mathrm{a}, 4} r_{\mathrm{a}, 2}^{-1} r_{2,-2}^{-\frac{7}{4}} r_{1,10}^{\frac{1}{4}} \rho^{-1} .
$$

Though this fiducial value would require long exposures on today's best instruments ${ }^{4}$, the uncertainty in the disk parameters allows a significant increase in fluence. Furthermore, the stong sensitivity to the temperature of the neutron star could allow for disks to be detected surrounding younger, hotter NSs.

\subsection{Radio Disk Detection}

Under suitable conditions, an asteroid belt may be detected through reflections of pulsed radio emission from asteroids larger than the wavelength (Phillips 1993). The ratio of reflected to pulsed flux is approximately

$$
\begin{aligned}
\frac{S_{u}}{S_{p}} & =\frac{3 M_{a} A}{4 \pi r^{2} \rho} f_{g} \tilde{R}_{\mathrm{a}}^{-1} \\
\tilde{R}_{a} & =\frac{\left\langle R_{a}^{3}\right\rangle}{\left\langle R_{a}^{2}\right\rangle},
\end{aligned}
$$

where $f_{g}$ is a geometrical factor that takes into account beaming of the pulsed flux and the solid angle into which pulsed flux is reflected; $\left\langle R_{a}^{x}\right\rangle$ is the average over a distribution of asteriod sizes for sizes larger than the wavelength, implying that

\footnotetext{
${ }^{4}$ The Spitzer IRAC point-source sensitivity is $0.6 \mu \mathrm{Jy}(1 \sigma)$ for a $100 \mathrm{~s}$ exposure at $3.6 \mu \mathrm{m}$ (http://ssc.spitzer.caltech.edu/irac//
} 
the mass $M_{a}$ in the belt also includes only those objects. In his analysis, Phillips (1993) assumed a fan beam for the pulsar radiation with $f_{g}=1$; for many pulsars, a pencil beam appears to be more appropriate, leading to $f_{g}<1$. For nominal parameters we have

$$
\frac{S_{u}}{S_{p}}=10^{-5.2} \frac{f_{g} A_{0.1} M_{\mathrm{a},-4}}{\rho r_{10}^{2} \tilde{R}_{\mathrm{a}, \mathrm{km}}} .
$$

For strong pulsars with $S_{p} \approx 1 \mathrm{Jy}$, the unpulsed reflected flux $\sim 10 \mu \mathrm{Jy}$. With present instrumentation this is very difficult to measure while with the EVLA and the Square Kilometer Array, it will be straightforward to detect or to place highly constraining limits.

\subsection{High-energy Studies}

Canonical radio pulsars are easily detected in X-rays through their magnetospheric emission and, in some cases, their thermal surface radiation. For cases where an acceleration region is reactivated by an asteroid, as is plausibly the case for the RRAT objects, we may expect to also see highenergy radiation.

\subsection{Interstellar Comets}

While no interstellar comet (ISC) has yet been identified, the Oort cloud's existence implies that a large number of ISCs should exist (e.g. Stern 1990). A typical density may be $n_{c}=$ $10^{14} n_{14} \mathrm{pc}^{-3}$, so for a characteristic velocity of $30 \mathrm{~km} \mathrm{~s}^{-1}$ and a cross section for an asteroid disk of $\pi r_{2}^{2}=10^{-4} \pi r_{2,-2}^{2} \mathrm{AU}^{2}$, the typical collision time is

$$
t_{c}=10^{4.6} \mathrm{yr}\left(n_{14} r_{2,-2}^{2} v_{30}\right)^{-1},
$$

which is too long to be of interest for our standard disk. However, a dense disk extending to $r_{2} \sim 1 \mathrm{AU}$ and having high optical depth would show an event about once per year. Comet-disk collisions would be brief but might induce highly episodic behavior in some objects.

\subsection{Pulsar Dependent Disk Properties}

The accretion model can be used as the basis for inferring the presence and properties of disks from the emission properties of various objects. Time-variable effects appear in some objects and not others, suggesting that there is a wide range of disk masses among pulsars and a wide range in instrinsic pulsar properties that regulate the inflow of neutral material. A variation in disk properties is not surprising because pulsars comprise a runaway stellar population whose velocities originate from momentum kicks imparted at or shortly after the formation of the NS (e.g. Lai et al. 2001). The amount of fallback material that ultimately settles into a disk will depend on the amplitude of the natal kick. A correlation study of NS space velocities and their radio intermittency may provide additional confirmation of the model and constraints on disk properties.

\section{SUMMARY AND CONCLUSIONS}

We have presented a new model that highlights the effects that asteroids in circumpulsar disks will have on electrodynamic and radiation processes in the magnetospheres of neutron stars. Implied phenomena may occur in both active radio pulsars and those that are usually quiescent. The key element is the injection of asteriods as macroscopic objects into pulsar magnetospheres, whereupon they evaporate and provide charges that are further processed. The rich set of consequences of this picture unifies what is otherwise a dizzying array of phenomena - drifting subpulses, pulse-shape mode changes, nulling, and bursts. Support for the model includes the theoretical expectation that orbital debris will accumulate in supernova fallback disks and that large asteroids can survive for much longer than the typical radio lifetime of a radio pulsar. Collisions and radiation-driven migration - processes identified in the solar system — inject asteriods into the light cylinder and provide charges at rates that perturb acceleration regions and presumably radio-emission. Objects that show bursts or termination of emission (nulls) with short rise and fall times appear to have spin periods and period derivatives that are consistent with objects that are expected to be cool and have large magnetospheres, just those for which asteroidal contamination is the most likely. Empirical confirmation of the model would involve detection of debris disks around pulsars that show intermittency and mass-constraining nondetections for those that do not. Unfortunately, disk detection is unlikely with existing instrumentation and may require the EVLA and the SKA to detect reflected pulsar radiation in the radio band or a large infrared instrument for direct detection. The quasi-periodic burster, B1931+24, may provide the best opportunity with existing telescopes because the elliptical orbit in our model suggests the presence of additional objects that may produce orbital recoil of the pulsar measureable in pulse timing data.

Further studies are warranted to explore asteroidal induced torque variations and timing noise, inference of disk properties from radio-emission properties, multiwavelength studies of objects that do and do-not show associated radio phenomena, and theoretical study of fallback disks as a function of supernova kick amplitudes. Correlation studies will test the model and point to other information-gleaning methods, such as exploring the relationship between the spin/magneticmoment angle and the occurrence of nulls and finding exceptional objects, such as young, hot neutron stars that show nulls and appear to contradict the model. The spin history may determine which objects retain asteroid belts, as those NS born with high magnetic field strengths and high spin rates will significantly ablate asteroids. However, a dense disk may rapidly spin down the NS and allow asteroids to form. The durations of nulls and bursts are closely related to the lifetimes of asteroids as they enter a pulsar magnetosphere. The lifetime, in turn, depends on radiative and particle conditions within the magnetosphere, so observable effects may provide robust contraints on NS temperatures, radiation mechanisms, particle flows, and NS masses via cooling mechanisms that are mass dependent.

We thank Joe Burns, Michael Kramer, Richard Lovelace, Andrew Lyne, Maura McLaughlin, Marina Romanova, and Ira Wasserman for helpful conversations. This work was supported by the National Science Foundation through grant AST-02-06035 to Cornell University. 
Arzoumanian, Z., Chernoff, D. F. \& Cordes, J. M., 2002, ApJ, 356, 289

Asseo, E. \& Melikidze, G. I. 1998, MNRAS, 301, 59

Becker, W., \& Trümper, J. 1997, A\&A, 326,382

Biggs, J. D. 1992, ApJ, 394, 574

Blackman, E. G., \& Perna, R. 2004, ApJ, 601, L71

Bryden, G., Beichman, C. A., Rieke, G. H., Stansberry,J. A, Stapelfeldt,K. R., Trilling, D. E.,Turner, N. J., Wolszczan,A. astro-ph/0604115

Burns, J. A., Lamy, P. L., \& Soter, S. 1979, Icarus, 40, 1

Cairns, I. H., ApJ, 610,948

Cheng, A. F., \& Ruderman, M. A. 1980, ApJ, 235, 576

Cheng, A. F. 1985, ApJ, 299, 917

Cheng, K. S., Ho, C., \& Ruderman, M. 1986, ApJ, 300, 522

Cheng, K. S., Ruderman, M., \& Zhang, L. 2000, ApJ, 537, 964

Chesley, S. R., Ostro, S. J., Vokrouhlicyý, D., Čapek, D., Giorgini, J. D. Nolan, M. C., Margot, J. L., Hine, A. A., Benner, L. A.M. \& Chamberlin, A. B. 2003, Science, 3025651

Coddington, I., Engels, P, Schweikhard, V. \& Cornell, E. A. 2003, Phys. Rev. Lett., 9110, 402

Cognard, I., Shrauner, J. A., Taylor, J. H., \& Thorsett, S. E. 1996, ApJ, 457, L81

Colgate, S. A. \& Petschek, A. G. 1981, ApJ, 348, 771

Cordes, J. M., \& Helfand, D. J. 1980, ApJ, 239, 640

Cordes, J. M., \& Greenstein, G. 1981, ApJ, 245, 1060

Cordes, J. M., \& Downs, G. S. 1985, ApJS, 59, 343

Cordes, J. M. 1993, ASP Conf. Ser. 36: Planets Around Pulsars, 36, 43

Cordes, J. M., Bhat, N. D. R., Hankins, T. H., McLaughlin, M. A., \& Kern, J. 2004, ApJ, 612, 375

Cordes, J. M., et al. 2006, ApJ, 637, 446

Deich, W. T. S., Cordes, J. M., Hankins, T. H., \& Rankin, J. M. 1986, ApJ, 300,540

Deshpande, A. A., \& Rankin, J. M. 1999, ApJ, 524, 1008

Dohnanyi, J. W. 1969, JGR, 74, 2531

Dyks, J., Rudak, B., \& Harding, A. K. 2004, ApJ, 607, 939

Ekşi, K. Y., \& Alpar, M. A. 2005, ApJ, 620, 390

Everett, J. E. \& Weisberg J. M. 2001, ApJ, 553,341

Faulkner, A. J., et al. 2004, MNRAS, 355, 147

Gogoberidze, G., Machabeli, G. Z., Melrose, D. B., \& Luo, Q. 2005, MNRAS, 360, 669

Goldreich, P. \& Julian, W. H. 1969, ApJ, 157, 869

Goldreich, P. \& Tremaine, S. 1978, Icarus, 34,227

Goldreich, P., Lithwick, Y., \& Sari, R. 2004, ApJ, 614, 497

Hankins, T. H., Kern, J. S., Weatherall, J. C., \& Eilek, J. A. 2003, Nature, 422, 141

Herbert, F. 1989, Icarus, 78, 402

Hirotani, K., Harding, A. K. \& Shibata, S. 2003, ApJ, 591334

Hobbs, G., Lyne, A. G., Kramer, M., Martin, C. E., \& Jordan, C. 2004 MNRAS, 353, 1311

Illarionov, A. F. \& Sunyaev, R. A., 1975, A.\&A, 39, 185

Jessner, A., Lesch, H., \& Kunzl, T. 2001, ApJ, 547, 959

Jenet, F. A., Anderson, S. B. \& Prince, T. A. 2001, ApJ, 546, 394

Jiang, Z.-B., \& Li, X.-D. 2005, Chinese Journal of Astronony and Astrophysics, 5, 487

Johnston, S., \& Romani, R. W. 2003, ApJ, 590, L95

Jones, P. B. 1982, MNRAS, 200, 1081

Konacki, M., \& Wolszczan, A. 2003, ApJ, 591, L147

Kramer, M. et al. 2006, Science Express, 1124060, 1
Lai, D., Chernoff, D. F., \& Cordes, J. M. 2001, ApJ, 549, 1111

Lewandowski, W., Wolszczan, A., Feiler, G., Konacki, M., \& Sołtysiński, T. 2004, ApJ, 600, 905

Lorimer, D. R., Camilo, F., \& Xilouris, K.M. 2002 AJ, 123, 1750

Lu, Y., \& Cheng, K. S. 2002, Chinese Journal of Astronony and Astrophysics, 2,161

McLaughlin, M. A., et al. 2006, Nature, 439, 817

Menou, K., Perna, R., \& Hernquist, L. 2001, ApJ, 559, 1032

271

Michel, F. C., \& Dessler, A. J. 1981, ApJ, 251, 654

Miller, M. C. \& Hamilton, D. P. 2001, ApJ, 550, 863

Mitra, D. \& Rankin, J. M. 2002, ApJ, 577,322.

Murray, C. D., \& Dermott,S. F., 1999, Solar System Dynamics

Muslimov, A. \& Harding, A. K. 2003, ApJ, 438, 314

Nesvorný, D., \& Bottke, W. F. 2004, Icarus, 170, 324

Nice, D. J., Splaver, E. M., Stairs, I. H., Löhmer, O., Jessner, A., Kramer, M. \& Cordes, J. M. 2005, ApJ, 634, 1242

Peale, S. J., Cassen, P. \& Reynolds, R. T. 1979, Science, 203, 892

Phillips, J. A. 1993, ASP Conf. Ser. 36: Planets Around Pulsars, 36, 321

Rafikov, R. R. 2003, AJ, 126, 2529

Rankin, J. M. 1990, ApJ 352,247

Rankin, J. M. 1993, ApJS,85,145

Raup, D. M. 1986, New York, N.Y. : Norton, c1986. 1st ed.,

Redman, S. L., Wright, G. A. E., \& Rankin, J. M. 2005, MNRAS, 357, 859

Reynolds, S. P. et al. 2006, ApJ, 639, 71

Richardson, D. C., Leinhardt,Z. M., Melosh, H. J., Bottke Jr.,W. F., Asphaug,E. 2002. In Bottke,Jr. W. F., Cellino,A., Paolicchi,P., Binzel,R. P. (Eds.), Asteroids III.

Romani, R. W. \& Yadigaroglu, I.-A. 1995, ApJ, 438, 314

Ruderman, M. A., \& Sutherland, P. G. 1975, ApJ, 196, 51

Rybicki, G. B., \& Lightman, A. P. 1986, Radiative Processes in Astrophysics, Wiley-VCH

Scharlemann, E. T., Arons, J., \& Fawley, W. M. 1978, ApJ, 222, 297

Sigurdsson, S., Richer, H. B., Hansen,B. M., Stairs I. H., Thorsett, S. .E. 2003, Science, 301,193

Slane, P. O., Helfand, D. J., \& Murray, S. S. 2002, ApJ, 571, L45

Sonett, C. P. et al. 1970, Ap. Sp. Sci., 7, 446

Stern, S. A. 1990, PASP, 102, 793

Stewart, G. R., Lin D. N. C., \& Bodenheimer P., in "Compact Stellar X-ray

Sources", Eds. R. Greenberg and A. Brahic, 1984

Takata, J. et al. 2004, MNRAS, 354, 1120

Takata, J. et al. 2006, MNRAS, 366, 1310

Thorsett, S. E., Phillips, J. A., \& Cordes, J. M. 1993, ASP Conf. Ser. 36 Planets Around Pulsars, 36, 31

Usov, V. V. 1987, ApJ, 320333

Vivekanand, M. 1995, MNRAS, 274, 785

Vivekanand, M., \& Narayan, R. 1981, Journal of Astrophysics and Astronomy, 2, 315

Voit, G. M. 1991, ApJ, 379, 122

Wang, Z., Chakrabarty, D. \& Kaplan, D. L. 2006, Nature, 440, 772

Wright, G. A. E. 2003, MNRAS, 344, 1041

Yakovlev, D. G., \& Pethick, C. J. 2004, ARA\&A, 42, 169

Zhang, L., Cheng, K. S., Jiang, Z. J., \& Leung, P. 2004, ApJ, 604, 317

Zhang, B., Gil, J. \& Dyks, D. 2006, astro-ph/0601063

\section{APPENDIX}

\section{INDUCTION HEATING}

We consider two types of induction heating, one from the temporally changing magnetic field experienced by an asteroid that has no net current flow through it, the other from a field-induced current through the asteroid.

Well outside the corotation radius, $r_{c o}=\left[G M_{*} P^{2} /(2 \pi)^{2}\right]^{1 / 3} \approx 10^{8.2} \mathrm{~cm} P^{2 / 3}$, the asteroid is subjected to a changing magnetic field, $\partial B / \partial t$, that is determined by the NS spin; this tends to zero as the asteroid approaches corotation but most asteroids will evaporate before reaching corotation. The generated electric field $\propto \partial B / \partial t$ in the asteroid's frame is dissipated within a skin depth $\delta \ll R_{a}$, yielding an equilibrium temperature (with $\sigma$ the conductivity and $\sigma_{\mathrm{SB}}$ the Stefan-Boltzmann constant)

$$
T_{a, \sigma}=\left(\frac{c}{24 \pi \sigma_{\mathrm{SB}}}\right)^{1 / 4}(P \sigma)^{-1 / 8} B^{1 / 2} \approx 10 K P^{-1 / 8}\left(B_{12} \sin \alpha\right)^{1 / 2} r_{10}^{-3 / 2}\left(\frac{\sigma}{\sigma_{\max }}\right)^{-1 / 8},
$$

where $\sigma / \sigma_{\max }$ is the conductivity relative to a maximum that is taken to be that of pure copper, $\sigma_{\mathrm{Cu}} \approx 5 \times 10^{17} \mathrm{~s}^{-1}$. Asteroidal material will have lower conductivities and thus higher temperatures than implied in the equation. However, the skin depth $\delta=(c / 2 \pi) \sqrt{P / \mu \sigma}(\sim 7 \sqrt{P} \mathrm{~cm}$ for copper) also increases with decreasing conductivity so that an asteroid's resistance reaches a maximum when $\delta \sim R_{\mathrm{a}}$. This limits the heating and thus the temperature coefficient to only a factor of a few larger than in Eq. A1 Ohmic heating increases faster with decreasing radius than radiative heating and would be competitive with radiative heating at radii $\sim 10^{8} \mathrm{~cm}$ if asteroids could reach that radius (and ignoring corotation).

The electric field in the frame of an asteroid moving orbitally or ballistically near the light cylinder of a pulsar with $v \ll c$, is $\mathbf{E}=(\Omega \times \mathbf{r}) \times \mathbf{B} / c$, or approximately $10^{5.4}$ volts $\mathrm{m}^{-1} B_{12}\left(r_{\mathrm{LC}} / r\right)^{2} P^{-3} R_{*, 6}^{3}$. If a circuit is established through the magnetosphere and asteroid, the current density $\mathbf{J}=\sigma \mathbf{E}$ yields ohmic heating power $\tilde{P} \approx R_{a}^{3} J E$ as long as the induced azimuthal magnetic field 
at the asteroid surface satisfies $B_{i} \approx 2 \pi R_{a} J / c \lesssim B$. The implied maximum current density, $J_{\max } \approx c B / 2 \pi R_{a}$, corresponds to an evaporation flux,

$$
\frac{d \dot{N}}{d A}=\frac{J_{\max }}{e} \approx 10^{18.0} \mathrm{~s}^{-1} \mathrm{~cm}^{-2} B_{12} P^{-3} R_{*, 6}^{3} R_{a, 2}^{-1}\left(r_{\mathrm{LC}} / r\right)^{3} .
$$

and a circuit resistance $\sim 4 r / c r_{\mathrm{LC}}$ (cgs) or about $120\left(r / r_{\mathrm{LC}}\right)$ Ohms. The maximum heating power is

$$
\tilde{P}_{\max } \approx c\left(R_{a} B\right)^{2}\left(r / r_{\mathrm{LC}}\right) \approx 10^{16.4} \mathrm{erg} \mathrm{s}^{-1}\left[P^{-3} B_{12} R_{a, 2} R_{*, 6}^{3}\right]^{2}\left(r_{\mathrm{LC}} / r\right)^{5} .
$$

When the current density is less than $J_{\max }$, the heating rate is $\tilde{P}=\left(J / J_{\max }\right) \tilde{P}_{\max }$. The corresponding equilibrium temperature is

$$
T_{a, i} \approx\left(\frac{\tilde{P}}{4 \pi \sigma_{\mathrm{SB}} R_{a}^{2}}\right)^{1 / 4} \approx 10^{3.9} \mathrm{~K}_{a, 2}^{-1 / 2}\left(\frac{\tilde{P}_{\max }}{10^{16.4} \mathrm{erg} \mathrm{s}^{-1}}\right)^{1 / 4}\left(\frac{\tilde{P}}{\tilde{P}_{\max }}\right)^{1 / 4} .
$$

As an asteroid first enters the magnetosphere at $r \sim r_{\mathrm{LC}}$ of a long-period pulsar, it is too cool to provide the necessary particle flux (c.f. Figure 5), so heating is dominated by radiative effects (see text) until evaporation becomes large enough to supply the current. For NS with $10^{5} \mathrm{~K}$ surface temperatures and low magnetic fields, injected asteroids reach $\sim 10^{9} \mathrm{~cm}$ before they have $2000 \mathrm{~K}$ temperatures (Eq. 18 and Figure 4), at which their evaporation rates are too low to provide the full current density. However, with a canonical field strength of $10^{12} \mathrm{G}$, the asteroid temperature and evaporation rise very rapidly with decreasing radius so that runaway heating drives an evaporation avalanche, exploding the asteroid. This occurs at about $10^{9.3} \mathrm{~cm}$ for a metersized asteroid. For pulsars with smaller field strengths, this radius will decrease. These numbers are altered if there is significant particle flux from elsewhere in the magnetosphere that provides a significant amount of current through the asteroid. 
TABLE A 1

PARAMETERS For Nulling AND NULling-Limited PUlsars

\begin{tabular}{|c|c|c|c|c|c|c|c|}
\hline Name (J2000) & Name (B1950) & $\begin{array}{l}P \\
(\mathrm{~s})\end{array}$ & $\begin{array}{c}\log \dot{P} \\
\left(\mathrm{~s} \mathrm{~s}^{-1}\right)\end{array}$ & $\begin{array}{c}\log \tau_{s} \\
(\mathrm{yr})\end{array}$ & $\begin{array}{l}\text { Nulling Fraction } \\
(\%)\end{array}$ & $\begin{array}{c}\alpha \\
(\mathrm{deg})\end{array}$ & References \\
\hline J1744-3922 & & 0.1724 & -17.8 & 9.25 & $75 \pm 32$ & $\ldots$ & F04, $\cdots$ \\
\hline J0828-3417 & B0826-34 & 1.8489 & -15.0 & 7.48 & $70 \pm 35$ & 5.0 & B92, Upper \\
\hline J1944+1755 & B1942+17 & 1.9969 & -15.1 & 7.64 & 60 & $\ldots$ & B92, $\cdots$ \\
\hline $\mathrm{J} 1115+5030$ & $\mathrm{~B} 1112+50$ & 1.6564 & -14.6 & 7.02 & $60 \pm 5$ & 32 & B92, R93 \\
\hline $\mathrm{J} 1819+1305$ & $\ldots$ & 1.0604 & -15.4 & 7.67 & 50 & $\cdots$ & $\mathrm{LCX} 02, \cdots$ \\
\hline J1946+1805 & B1944+17 & 0.4406 & -16.6 & 8.46 & $50 \pm 7$ & $\ldots$ & V95, R93 \\
\hline J0034-0721 & B0031-07 & 0.9430 & -15.4 & 7.56 & $44.6 \pm 1.3$ & 6 & V95, R93 \\
\hline J0754+3231 & B $0751+32$ & 1.4423 & -15.0 & 7.33 & $34 \pm 0.5$ & 26 & B92, R93 \\
\hline $\mathrm{J} 1649+2533$ & $\ldots$ & 1.0153 & -15.3 & 7.46 & 30 & $\ldots$ & $\mathrm{L} 04, \cdots$ \\
\hline $\mathrm{J} 0528+2200$ & B $0525+21$ & 3.7455 & -13.4 & 6.17 & $25 \pm 5$ & 21 & B92, MR02 \\
\hline $\mathrm{J} 2321+6024$ & B $2319+60$ & 2.2565 & -14.2 & 6.71 & $25 \pm 5$ & 18 & B92, R93 \\
\hline J1945-0040 & B1942-00 & 1.0456 & -15.3 & 7.49 & $21 \pm 1$ & 26 & B92, R93 \\
\hline $\mathrm{J} 1136+1551$ & B $1133+16$ & 1.1880 & -14.4 & 6.70 & $15.0 \pm 2.5$ & 46 & B92, MR02 \\
\hline $\mathrm{J} 2113+4644$ & B $2111+46$ & 1.0147 & -15.1 & 7.35 & $12.5 \pm 2.5$ & 9 & B92, R93 \\
\hline J0942-5552 & B0940-55 & 0.6644 & -13.6 & 5.66 & $\leq 12.5$ & 25 & B92, R93 \\
\hline J2330-2005 & B2327-20 & 1.6436 & -14.3 & 6.75 & $12 \pm 1$ & 60 & B92, R90 \\
\hline $\mathrm{J} 0659+1414$ & B $0656+14$ & 0.3849 & -13.3 & 5.05 & $12 \pm 4$ & 30.0 & B92, R93 \\
\hline $\mathrm{J} 1057-5226$ & B1055-52 & 0.1971 & -14.2 & 5.73 & $\leq 11$ & 90.0 & B92, B93 \\
\hline J0304+1932 & B $0301+19$ & 1.3876 & -14.9 & 7.23 & $10 \pm 5$ & 38 & B92, MR02 \\
\hline $\mathrm{J} 2305+3100$ & $\mathrm{~B} 2303+30$ & 1.5759 & -14.5 & 6.93 & $10 \pm 3$ & 20.5 & RWR05, R93; \\
\hline $\mathrm{J} 1900-2600$ & B1857-26 & 0.6122 & -15.7 & 7.68 & $10 \pm 2.5$ & 25 & B92, R93 \\
\hline J2048-1616 & B2045-16 & 1.9616 & -14.0 & 6.45 & $10.0 \pm 2.5$ & 34 & V95, MR02 \\
\hline J0943+1631 & B0940+16 & 1.0874 & -16.0 & 8.28 & $8 \pm 3$ & 53 & B92, R93 \\
\hline $\mathrm{J} 2157+4017$ & B $2154+40$ & 1.5253 & -14.5 & 6.85 & $7.5 \pm 2.5$ & 20 & B92, R93 \\
\hline $\mathrm{J} 0837+0610$ & B $0834+06$ & 1.2738 & -14.2 & 6.47 & $7.1 \pm 0.1$ & 30 & B92, MR02 \\
\hline J0944-1354 & B0942-13 & 0.5702 & -16.3 & 8.30 & $\leq 7$ & 45 & V95, R93 \\
\hline $\mathrm{J} 1532+2745$ & B $1530+27$ & 1.1248 & -15.1 & 7.36 & $6 \pm 2$ & 26 & B92, R93 \\
\hline $\mathrm{J} 1239+2453$ & B $1237+25$ & 1.3824 & -15.0 & 7.36 & $6.0 \pm 2.5$ & 53 & B92, MR02 \\
\hline J0151-0635 & B0148-06 & 1.4647 & -15.4 & 7.72 & $\leq 5$ & 14.5 & B92, R93 \\
\hline $\mathrm{J} 2022+5154$ & $\mathrm{~B} 2021+51$ & 0.5292 & -14.5 & 6.44 & $\leq 5$ & 23 & B92, R93 \\
\hline $\mathrm{J} 1926+0431$ & B1923+04 & 1.0741 & -14.6 & 6.84 & $\leq 5$ & 34.5 & B92, R93 \\
\hline $\mathrm{J} 1614+0737$ & B1612+07 & 1.2068 & -14.6 & 6.91 & $\leq 5$ & 24.5 & B92, R93 \\
\hline $\mathrm{J} 0953+0755$ & B0950+08 & 0.2531 & -15.6 & 7.24 & $\leq 5$ & 74.6 & V95, EW01 \\
\hline $\mathrm{J} 1841+0912$ & B1839+09 & 0.3813 & -15.0 & 6.74 & $\leq 5$ & 86.1 & B92, EW01 \\
\hline J0826+2637 & B $0823+26$ & 0.5307 & -14.8 & 6.69 & $\leq 5$ & 81.1 & B92, EW01 \\
\hline $\mathrm{J} 1910+0358$ & B $1907+03$ & 2.3303 & -14.3 & 6.92 & $4.0 \pm 0.2$ & 6.0 & B92, R90 \\
\hline $\mathrm{J} 1243-6423$ & B1240-64 & 0.3885 & -14.3 & 6.14 & $\leq 4$ & 33 & B92, R93 \\
\hline J1456-6843 & B1451-68 & 0.2634 & -16.0 & 7.63 & $\leq \overline{3} .3$ & 37.0 & B92, R93 \\
\hline $\mathrm{J} 2317+2149$ & B $2315+21$ & 1.4447 & -15.0 & 7.34 & $3.0 \pm 0.5$ & 88 & B92, R93; \\
\hline $\mathrm{J} 2022+2854$ & $\mathrm{~B} 2020+28$ & 0.3434 & -14.7 & 6.46 & $\leq 3$ & 56 & $\mathrm{~B} 92, \mathrm{MR02}$ \\
\hline J0152-1637 & B0149-16 & 0.8327 & -14.9 & 7.01 & $\leq 2.5$ & 84 & V95, R93 \\
\hline $\mathrm{J} 2219+4754$ & B $2217+47$ & 0.5385 & -14.6 & 6.49 & $\leq 2$ & 42 & B92, R93 \\
\hline J0814+7429 & B $0809+74$ & 1.2922 & -15.8 & 8.09 & $1.42 \pm \overline{0.02}$ & 9 & B92, R93 \\
\hline J0837-4135 & B0835-41 & 0.7516 & -14.5 & 6.53 & $\leq 1.2$ & 50 & B92, R93 \\
\hline J0820-1350 & B0818-13 & 1.2381 & -14.7 & 6.97 & $1.01 \pm 0.01$ & 15.5 & B92, R93 \\
\hline $\mathrm{J} 1932+1059$ & B1929+10 & 0.2265 & -14.9 & 6.49 & $\leq 1$ & 35.97 & B92, EW01 \\
\hline $\mathrm{J} 2116+1414$ & B2113+14 & 0.4402 & -15.5 & 7.38 & $\leq 1$ & 30.0 & B92, R93 \\
\hline $\mathrm{J} 1820-0427$ & B1818-04 & 0.5981 & -14.2 & 6.18 & $\leq \overline{0.75}$ & 65 & B92, R93 \\
\hline $\mathrm{J} 1948+3540$ & B1946+35 & 0.7173 & -14.2 & 6.21 & $\leq 0.75$ & 32.0 & B92, R90 \\
\hline $\mathrm{J} 1752-2806$ & B1749-28 & 0.5626 & -14.1 & 6.04 & $\leq 0.75$ & 42 & B92, R93 \\
\hline $\mathrm{J} 2055+3630$ & B $2053+36$ & 0.2215 & -15.4 & 6.98 & $\leq 0.7$ & 34 & B92, R93 \\
\hline J0452-1759 & B0450-18 & 0.5489 & -14.2 & 6.18 & $\overline{\leq} 0.5$ & 24.0 & B92, R93 \\
\hline J1913-0440 & B1911-04 & 0.8259 & -14.4 & 6.51 & $\overline{\leq} 0.5$ & 64.0 & B92, R93 \\
\hline $\mathrm{J} 1823+0550$ & B $1821+05$ & 0.7529 & -15.6 & 7.72 & $\overline{\leq} 0.4$ & 32 & B92, R93 \\
\hline J0738-4042 & B $0736-40$ & 0.3749 & -14.8 & 6.57 & $\leq 0.4$ & 17.0 & B92, R93 \\
\hline J1644-4559 & B1641-45 & 0.4551 & -13.7 & 5.56 & $\leq 0.4$ & 33 & B92, R93 \\
\hline J0630-2834 & B0628-28 & 1.2444 & -14.1 & 6.44 & $\overline{\leq} 0.3$ & 13.5 & B92, R93 \\
\hline $\mathrm{J} 2018+2839$ & $\mathrm{~B} 2016+28$ & 0.5580 & -15.8 & 7.78 & $\leq 0.25$ & 39 & B92, R93 \\
\hline $\mathrm{J} 1921+2153$ & B1919+21 & 1.3373 & -14.9 & 7.20 & $\overline{\leq} 0.25$ & 34 & B92, MR02 \\
\hline $\mathrm{J} 1534-5334$ & B1530-53 & 1.3689 & -14.9 & 7.18 & $\leq 0.25$ & $\cdots$ & B92, PCE \\
\hline $\mathrm{J} 0332+5434$ & B $0329+54$ & 0.7145 & -14.7 & 6.74 & $\leq 0.25$ & 32 & B92, MR02 \\
\hline
\end{tabular}


TABLE A1 - Continued

\begin{tabular}{|c|c|c|c|c|c|c|c|}
\hline Name (J2000) & Name (B1950) & $\begin{array}{l}P \\
(\mathrm{~s})\end{array}$ & $\begin{array}{c}\log \dot{P} \\
\left(\mathrm{~s} \mathrm{~s}^{-1}\right)\end{array}$ & $\begin{array}{c}\log \tau_{s} \\
(\mathrm{yr})\end{array}$ & $\begin{array}{c}\text { Nulling Fraction } \\
(\%)\end{array}$ & $\begin{array}{c}\alpha \\
(\mathrm{deg})\end{array}$ & References \\
\hline $\mathrm{J} 1645-0317$ & B1642-03 & 0.3877 & -14.7 & 6.54 & $\leq 0.25$ & 70 & B92, R93 \\
\hline J0742-2822 & В0740-28 & 0.1668 & -13.8 & 5.20 & $\leq 0.2$ & 37 & B92, R93 \\
\hline $\mathrm{J} 1844+1454$ & B1842+14 & 0.3755 & -14.7 & 6.50 & $\overline{\leq 0.15}$ & 29 & B92, R90 \\
\hline $\mathrm{J} 1919+0021$ & B1917+00 & 1.2723 & -14.1 & 6.42 & $\leq 0.1$ & 81 & B92, R93 \\
\hline $\mathrm{J} 1745-3040$ & B1742-30 & 0.3674 & -14.0 & 5.74 & $\leq 0.1$ & 51 & B92, R93 \\
\hline $\mathrm{J} 1731-4744$ & B1727-47 & 0.8298 & -12.8 & 4.91 & $\overline{\leq} 0.1$ & 64 & B92, R93 \\
\hline $\mathrm{J} 1607-0032$ & B1604-00 & 0.4218 & -15.5 & 7.34 & $\overline{\leq} 0.1$ & 50 & B92, MR02 \\
\hline $\mathrm{J} 0823+0159$ & B0820+02 & 0.8649 & -16.0 & 8.12 & $\leq 0.06$ & 46 & B92, R93 \\
\hline $\mathrm{J} 0525+1115$ & B $0523+11$ & 0.3544 & -16.1 & 7.88 & $\overline{\leq} 0.06$ & 78 & B92, R93 \\
\hline $\mathrm{J} 1935+1616$ & B1933+16 & 0.3587 & -14.2 & 5.98 & $<0.06$ & 72.0 & B92, R93 \\
\hline $\mathrm{J} 1430-6623$ & B1426-66 & 0.7854 & -14.6 & 6.65 & $\overline{\leq} 0.05$ & $\cdots$ & B92, PCE \\
\hline $\mathrm{J} 0922+0638$ & В0919+06 & 0.4306 & -13.9 & 5.70 & $\overline{\leq} 0.05$ & 48.0 & B92, R93 \\
\hline $\mathrm{J} 2046+1540$ & B2044+15 & 1.1383 & -15.7 & 8.00 & $\overline{\leq} 0.04$ & 40 & B92, R93 \\
\hline J1559-4438 & B1556-44 & 0.2571 & -15.0 & 6.60 & $\overline{\leq} 0.04$ & 32 & B92, R93 \\
\hline $\mathrm{J} 1740+1311$ & B $1737+13$ & 0.8031 & -14.8 & 6.94 & $\leq 0.02$ & 41 & B92, R93 \\
\hline $\mathrm{J} 0629+2415$ & B $0626+24$ & 0.4766 & -14.7 & 6.58 & $\overline{\leq} 0.02$ & 30 & B92, R93 \\
\hline J0437-4715 & & 0.0058 & -19.2 & 9.20 & $<\overline{0.0016}$ & $\ldots$ & B92, $\cdots$ \\
\hline J0835-4510 & B0833-45 & 0.089 & -12.9 & 4.05 & $\overline{\leq} 0.0008$ & 90 & B92, R93 \\
\hline
\end{tabular}

NotE. - A list of pulsars known to null and pulsars for which limits have been placed on nulling fraction. The period $P$, period derviative $\dot{P}$, the spin-down age $\tau_{s}$, and the angle between the spin and magnetic axes $\alpha$ are also given. The first reference is for the nulling fraction, and second is $\alpha$, the angle between the magnetic and spin axes. R90 refers to Rankin (1990), B92 to Biggs (1992), R93 to Rankin (1993), V95 to Vivekanand (1995), LCX02 to Lorimer et al. (2006), MR02 to Mitra \& Rankin (2002), EW01 to Everett \& Weisberg (2001), L04 to Lewandowski et al. (2004), F04 to Faulkner et al. (2004), and RWR05 to Redman et al. (2005) 